\title{
Quantifying Numerically Forecast Size Effects in the Free Vibration of Heterogeneous Beams
}

\author{
Bahman Hassanati ${ }^{1,}{ }^{*}$, Marcus Wheel ${ }^{1}$ \\ ${ }^{1}$ Mechanical \& Aerospace Engineering Department, University of Strathclyde, 75 Montrose Street, Glasgow G1 1XJ, \\ Scotland, UK \\ * Corresponding author: bahman.hassanati@strath.ac.uk
}

\begin{abstract}
This paper reports on the influence that a periodic microstructure has on the unconstrained flexural vibration of geometrically similar but differently sized heterogeneous beam samples. A numerical investigation was conducted by finite element analysis (FEA) incorporating the detailed heterogeneity to identify and quantify any effect of beam size on the transverse modal frequencies when the microstructural scale is comparable to the overall size. Finite element models of the macroscopic beam samples were created by firstly specifying microstructural scale unit cells containing a single void or inclusion using ANSYS Mechanical APDL and then repeatedly regenerating these as required. Four beam sizes consisting of one, two, three or four layers of unit cells were created while the length to depth aspect ratio was kept constant for all sizes. Void or inclusion volume fraction was also altered while keeping the homogenised mass and stiffness properties of each beam fixed. The influence of the beam boundary texture on the results was also investigated. The ANSYS results were compared to the analytical solution for a conventional Timoshenko beam and a nonlocal Timoshenko beam. Using the nonlocal Timoshenko analysis, the Eringen small length scale coefficients were estimated but found to be size dependent. Numerical predictions obtained from a novel control volume based finite element (CVFEM) procedure incorporating micropolar constitutive behaviour were therefore matched to the ANSYS results and thereby used to identify the two additional constitutive parameters featuring in planar micropolar elasticity theory, namely the characteristic length in bending and coupling number.
\end{abstract}

Keywords: Finite Element Analysis, Heterogeneous Material, Eringen's Micropolar Theory, Free Vibration, Modal Analysis, Beams' Dynamics

\section{Introduction}

The technological advances in recent decades such as in the aerospace, biomedical, nanotechnology industries and so forth, have created the need for the application of small-scale structures whose size is comparable to the microstructural length scale of the materials from which they are manufactured. This has created a whole new era for researchers to investigate the dynamic behaviour of structures where the classical theories of elasticity become increasingly invalid such as in the case of the flexural or transverse vibration of small-scale heterogeneous beams. Generally, heterogeneity is regarded as a discontinuity of physical properties of the material in either a specific direction or multi-directionally. In the literature, homogenisation methods are sought to represent the properties of materials comprised of periodic assemblies of a specified unit cell. Rabboh et al. [1] thus used the rule of mixtures to calculate the elastic constants and Poisson's ratio for functionally graded material sandwich beams and investigated the effect of the functional grading on the beams' dynamic behaviour. Della and Shu [2] used Eshelby's equivalent inclusion method to investigate the vibration of piezoelectric beams and their analytically obtained results indicate that a size effect arising from the size of piezoelectric inclusions, their location in the structure of the beam and their volume fraction is anticipated in their dynamic behaviour.

Homogenisation methods also become increasingly problematic when the size of constituent materials such as inclusions and/or voids becomes comparable to the overall size of the beam structure. Modifications to classical elasticity theories are only useful when the internal length scale parameters associated with the microstructure are considered very small. The size-dependent behaviour of materials has been reported by many researchers such as in the work by Groh \& Weaver [3], Gherlone [4], and Schulze et al. [5] in laminated beams. The results presented by Alghamdi and Dasgupta [6] in modelling active damping of adaptive structures show how the beam's time to decay varies as the device aspect ratio, inclusion shape, location and volume fraction is altered, and also show how changes 
in host stiffness result in changes in time to decay and electrical field. Timoshenko beam theory is widely used by researchers as it is able to account for rotary inertia and shear deformation and is therefore regarded as nonlocal if Eringen's small-scale effect [7] is incorporated in the governing equations. C M Wang et al. [8] used a finite segments method to calibrate Eringen's small length scale coefficient for initially stressed vibrating nonlocal beams and stated that ' $e_{0}$ does not depend on buckling or vibration modes'. Available results on the presence of size effects in the deformation of heterogeneous materials reported by researchers show deviation from elastic theories in static loading cases when the beam or plate L/h ratio reduces,e.g., [9]-[15]. Nakamura and Lakes [16] used a two dimensional FE method and investigated the localised end loads applied on a strip sample and concluded that as the characteristic length increases, the rate of decay of stress and strain energy reduces. The micropolar theory for example incorporates additional couple stresses and an associated degree of freedom, a microrotation and thus accounts for material size effects but requires the specification of additional constitutive parameters including a characteristic length. A 2D micropolar strip loaded at one end was investigated by Nakamura \& Lake [16], and the influences of elastic constants especially characteristic length and coupling number were studied. They concluded that for a significantly small characteristic length (in comparison with the strip's width), the rate of stress/or strain energy decreases as the characteristic length increases. In the dynamic case, this may be shown by wave dispersion. Their work predominantly included studying the models for various characteristic lengths and coupling numbers, $\mathrm{N}$, and provides no method to determine them. However in other work by Lakes [17], an extensive comparison was made between various theories, e.g. micropolar and Eringen's nonlocal theories. This shows that the elastic constants can be obtained by means of a dynamic wave propagation method. Nevertheless, in any field or wave based method that relies on determining size effects, there are limitations concerning the smallest characteristic length. Also, caution is required when the coupling number, $\mathrm{N}$, is close to its lower and upper bound values of zero and one when performing numerical analysis otherwise errors in computation may result.

Beveridge et al. [18] also studied the micropolar behaviour of perforated beams in the static 3 point bending case and determined the micropolar constants using test results and a control volume based finite element technique.

Waseem et al. [14] investigated the influence of void size on the constitutive properties of circularly shaped models containing voids (Perforated rings), and derived the final equation relating the stiffness to the specimen size by relating the diametrical loads, displacement and stored strain energy. They concluded that in models with smooth specimen surface (rings circumference), the stiffness changes linearly with sample size measure. McGregor [19] provides the same conclusions.

Wheel et al. [16] studied the influence of model size in heterogeneous beams when loaded in 3 point bending. They investigated size effects in beams with voids and showed that sample stiffness relates to the beam sample size, as measured by the reciprocal of its depth squared, in a linear manner. They also reported that there are both negative and positive effects of beam size depending on the beam boundary topology [15].

In section two of this paper, specific beam models with voids and inclusions of various volume fractions were modeled using the finite element method to account for the beam surface conditions. Then the non-dimensional modal frequencies $(\lambda)$ of beams with free-free boundary conditions, devoid of any external load, were investigated to ascertain the influence of any size effect. The normalized frequencies $(\Lambda)$ were also compared at each of the modal frequencies for particular beam models and volume fractions. Two widely used theories,, Eringen's non-local theory and micropolar theory, were studied with the aim of identifying whether they could explain the influence of the size effect seen in the dynamic behavior of non-homogeneous beams. These two theories incorporate length scale parameters in their formulations. Therefore, in section three, the Eringen's non-local theory was considered and formulated for a Timoshenko beam with free-free boundary conditions. Then the results from ANSYS FE and Eringen's non-local Timoshenko beams were compared, and shortcomings of the non-local theory for the beam models were highlighted. In section four, formulations based on micropolar theory was applied to the beam models, and a useful equation was derived which helps to identify one of the micropolar constants, namely characteristic length of bending, knowing the beams overall dimensions, and the primary modal frequency. Then an iteration method was employed to identify the other micropolar constant, namely the coupling number. For this reason, the frequencies from the CVFEM were compared with ANSYS finite element results and iterated upon to obtain the coupling number. Section 5 was dedicated to the development of the CVFEM code for free vibration problem of beams incorporating mass and micro inertia terms, and finally, micropolar beam models were modeled for further analysis. In section six, the use of the CVFEM code, for identification of the coupling number was described in more details and the shortcomings of this method for identifying the coupling number for some of the beam models were highlighted. Finally, the CVFEM predictions were compared with ANSYS FE results and discussed. 


\section{Finite Elements Analysis of Beams with Periodic Heterogeneity}

\subsection{Finite Element Modelling}

Three distinct types of 2D beams were modelled: those with perforations (voids), those with compliant inclusions and those with a compliant matrix. ANSYS APDL version 16.2 was used to perform modal analysis on each beam type. Figure 1 shows the two unit-cell configurations containing either voids or inclusions that were used to construct individual beam models. The height of the modelled unit cell is $0.866 \mathrm{~mm}$, and the length of the unit cells is $1 \mathrm{~mm}$ respectively. The void or inclusion centres are thus located on an equilateral triangular array. Models containing various void/inclusion volume fractions were generated using parametric APDL codes. The unit-cells consist of two isotropic materials so that inclusions are surrounded by matrix. Figure 1 shows unit-cells with inclusion of $0.2 \mathrm{~mm}$ in radius. For the unit cells of the beam models with continuous boundaries as seen in see figure 1, left, a quarter unit cell was first modeled and then reflected to produce a full unit cell. Therefore, all sides of the matrix section are divided by 12 parts and mapped so that the sizes of elements decrease on approaching the inclusion's border. The circular area containing the inclusion incorporates a squared area which is divided into a 12 by 12 element mesh and the remaining area is divided into two concentric rings. 8-node solid 183 elements were used for meshing the areas. By reflecting the quarter unit cell in both $\mathrm{x}$ and $\mathrm{y}$-directions, a complete unit cell was created which contained 1920 elements and 6068 nodes. To analyse the mesh convergence, beams with 28, 156, 528, 898, 1920 and 7680 elements per unit cells were modelled by changing the number of line divisions, and it was observed that the average percentage error of the frequencies of each model for the first ten modes compared with the next model refinement in sequence are $0.5674,0.0047,0.0002,0.0001$ and 0.0000 percent. Therefore, beams with 1920 elements per unit cell satisfied the requirements for mesh convergence for the beams with continuous boundaries. The beams with textured boundaries were modeled so that inclusions or voids were located at the center of a hexagonal matrix. The hexagon sides are divided into 12 equal parts. The diagonal lines connecting the hexagon's vertices to the circle are divided into ten parts. Such a mesh arrangement provides 1260 elements and 3925 nodes per unit cell. Beams with textured boundaries and 108, 276, 1260 and 5040 element/Unit-Cell were modeled and the average percentage error for the first ten modes reduced by $0.0078,0.0021$ and 0.0006 percent upon refinement. Therefore unit cells with 1260 elements have a $0.0006 \%$ different comparing with unit cells containing 5040 elements.

Void or inclusion radius was varied from 0.1 to $0.3 \mathrm{~mm}$ in $0.05 \mathrm{~mm}$ intervals. The corresponding void or inclusion volume fractions are listed in table 1 along with the equivalent radius normalised with respect to the unit cell height $\left(V_{r} / S_{y}\right)$, where $V_{r}$ is the void or inclusion radius, and $S_{y}$ is the height of the unit cell. Throughout this paper, void or inclusions radius, volume fraction and normalised radius are used interchangeably.

Table 1: Changes in void or inclusion volume fraction with radius and/or normalised radius of void or inclusion

\begin{tabular}{|ccc|}
$\begin{array}{c}\text { Void/inclusion } \\
\text { radius, } \mathbf{r} \\
\mathbf{m m}\end{array}$ & $\begin{array}{c}\text { Void/inclusion volume } \\
\text { fraction } \\
\%\end{array}$ & $\begin{array}{c}\text { Normalised } \\
\text { void/inclusion radius, } \\
\mathbf{V}_{\mathbf{r}} / \mathbf{S}_{\mathbf{y}}\end{array}$ \\
\hline 0.1 & $4 \%$ & 0.12 \\
\hline 0.15 & $8 \%$ & 0.17 \\
\hline 0.2 & $15 \%$ & 0.23 \\
\hline 0.25 & $23 \%$ & 0.29 \\
\hline 0.3 & $33 \%$ & 0.35 \\
\hline
\end{tabular}

In order to create the macroscopic scale beams, the unit cells were repeatedly regenerated to produce four different beam sizes consisting of one, two, three or four layers of cells through beam depth. The length, $L$, to depth, $d$, aspect ratio, (L/d) was kept constant at $10.4: 1$ so that all four sizes of the beam of a given volume fraction remained geometrically similar. Two variants of each beam were created: those based on the first unit cell contained boundaries comprised continuously of matrix material while those incorporating the second unit cell contained textured boundaries, see figure 2-a to 2-d.

When changing the volume fraction of each material the homogenised mass and stiffness properties of the beam were kept fixed. This enabled the size effect on the free vibration to be identified for various volume fractions when the unit cell mass and overall homogenised properties were kept constant. The aim is to investigate the frequency 
changes for various beam sizes and void/or inclusions volume fractions for lateral vibration modes. To achieve this, the ratio of modulus of elasticity of matrix to inclusion was set at 10:1 for the beams with compliant inclusions and 1:10 for the beams with the compliant matrix. The modulus of elasticity at the macroscopic scale remained $70 \mathrm{GPa}$, see appendix table 8 . The matrix and inclusions densities were also altered according to the void or inclusion volume fraction, as detailed in appendix table 9 while keeping the mass density of the unit cells constant at $2700 \mathrm{~kg} / \mathrm{m}^{3}$.

The material parameters were obtained using ANSYS FE, and the modal analysis results based on ANSYS FE were used as the basis for comparison in this paper. The ANSYS FE made it possible to easily model various beam types with the volume fractions and material constants associated with the constituent unit-cells being altered while keeping the properties of the equivalent homogenized beam unchanged. This approach resulted in identifying the influence of the size effect in the beam models which was not possible with other approaches.

\subsection{FE Results and Size Effect Predictions}

\subsubsection{Beams with continuous boundaries}

The ANSYS finite element results which are presented in this section and shown in figures 3 to 6 and tabulated in Appendix table 4 to 5 provide size effect information for beams with continuous surfaces. For this purpose, three types of beams with such surfaces are modelled and analysed:
1) Beams with voids
2) Beams with compliant inclusions
3) Beams with compliant matrix

Modal frequencies are non-dimensionalised using the equation (1):

$$
\lambda=L(2 \pi f)^{1 / 2}\left(\frac{12 \rho}{E d^{2}}\right)^{1 / 4}
$$

where $\lambda$ is the non-dimensional frequency parameter, $L$ is the beams length; $f$ is the numerically predicted flexural modal frequency in $\mathrm{Hz} ; \rho$ is the mass density; $E$ is the modulus of elasticity, and $d$ is the depth of the beam. The values for $\lambda$ for the first ten modal frequencies of homogeneous beams $\left(V_{r} / S_{y}=0\right)$ predicted using finite element analysis are compared to the analytically derived values based on Timoshenko beam theory in table 2 :

Table 2 : The non-dimensional modal frequencies $(\lambda)$ for homogeneous beams and with aspect ratio 10.4:1

\begin{tabular}{|cccc|}
\hline Mode & ANSYS FEA & Timoshenko & $\begin{array}{c}\text { CVFEM } \\
(\mathrm{Ib}=\mathbf{0}, \mathbf{N}=\mathbf{0}, \mathbf{k}=\mathbf{0}) * *\end{array}$ \\
\hline 1 & 4.655455 & 4.653041 & 4.6305153 \\
\hline 2 & 7.527076 & 7.516596 & 7.479576 \\
\hline 3 & 10.194069 & 10.171415 & 10.119781 \\
\hline 4 & 12.626892 & 12.590439 & 12.521737 \\
\hline 5 & 14.84537 & 14.795647 & 14.705318 \\
\hline 6 & 16.874623 & 16.813016 & 16.695666 \\
\hline 7 & 18.739625 & 18.667635 & 18.518158 \\
\hline 8 & 20.461702 & 20.380707 & 20.194872 \\
\hline 9 & 22.057553 & 21.968693 & 21.743882 \\
\hline 10 & 23.538186 & 23.441844 & 23.178677 \\
\hline
\end{tabular}

${ }^{* *} \mathrm{l} b$ is the characteristic length in bending, $\mathrm{N}$ is the coupling number, and $\mathrm{k}$ is micropolar constant, see section 4.2

The normalised values when presented in figures 3 onward were obtained by dividing the non-dimensional frequency parameter $(\lambda)$ at each modal frequency by its corresponding non-dimensional frequency from column two of table 2 , 
that is the ANSYS FEA derived values for $\lambda$. The $\lambda$ values in column 4 of table 2 derived by the CVFEM were used for normalisation of subsequent frequencies obtained from CVFEM as shown in figures 12 and 13.

Figure 3 shows how the normalised frequencies $(\Lambda)$ for the beams with voids and height of two unit-cells (Second smallest beam sample) vary with mode number. This behaviour is highly size dependent as seen in figure 4 for mode one. The homogeneous case is represented by the blue lines (dotted lines with solid square markers) and the FE results show that in this case, the normalised modal frequency is size independent. Appendix table 4 provides nondimensional frequency parameters $(\lambda)$ information for the first ten transverse vibration modes of beams with voids. For any given mode the size effect becomes more pronounced with diminishing beam size and is greatest for the smallest size of beams. The size effect is also more pronounced for beams with a higher void volume fraction. Interestingly, the size effect is apparently mode dependent; there is a distinct change in its nature such that after mode three changes in void radius causes a decrease rather than an increase in normalised frequencies $(\Lambda)$ as seen in figure 3. Results in appendix table 5 show that beams with compliant inclusions behave similarly.

In figure 4, the variations in frequency with size measure for the primary modes (flexural mode 1 ) are displayed in further detail. According to these results, it appears that variations in the normalised frequencies have an approximately linear relationship with the inverse of the square of the depth of the beam $\left(1 / d^{2}\right)$, except for the smallest beam sizes where the ratio of the radius of void or inclusion to beam thickness reaches the maximum.

The results for specimens with stiff inclusions and continuous surfaces show an entirely different dynamic behaviour, figure 5 shows changes in $\Lambda$ with mode number for the beams with height of two unit-cells (Second smallest beam sample). Here, a distinctly different size effect indicating that increasing volume fraction causes a decrease in $\Lambda$ at a given mode number for this sample size. Figure 6 shows the inverted size effect on normalised frequencies for the primary mode when the inclusions have a higher modulus of elasticity than the matrix. The size effect although inverted once more remains approximately linear across the three larger samples but again this does not extrapolate to the smallest sample size.

\subsubsection{Beams with textured boundaries}

If the boundaries of the beams are not continuous but textured due to their intersection with the voids or inclusions, there is a significant difference in dynamic behaviour. Beams with voids or compliant inclusions and textured boundaries showed similar behaviour as the beams with compliant matrix and continuous boundaries described already in section 2.2.1 and therefore are not discussed any further in this section.

Finally, beams with textured boundaries and a matrix comprised of compliant material with a lower modulus of elasticity exhibit a more conventional size effect with normalised frequency increasing as beam size reduces, appendix table 6 .

In summary, the numerical results shown in figures 3 to 6 and provided in tables 4 to 6 indicate that the forecast size effect depends on:

a) Beam depth

b) Void/inclusions volume fraction

c) The relative stiffness of matrix and inclusions

d) Beam surface topology

The remainder of this paper considers whether various analytical or numerical models incorporating size effects are capable of predicting these numerical results.

\section{An Analytical Nonlocal Timoshenko Beam Model}

\subsection{The Nonlocal Timoshenko beam Model}

In this section, the nonlocal Timoshenko (NLT) beam model is used to study the size effect in a Timoshenko beam by incorporating Eringen's nonlocal theory. This approach has been widely used in nanotechnology because it can be solved analytically for various boundary conditions, e.g., [7], [8], [20]. However, it must be noted that the Timoshenko beam theory is one dimensional and Eringen's small-scale coefficient is only really applicable to the longitudinal direction. 
The governing equations for nonlocal Timoshenko beam can be obtained by applying Hamilton's principle and incorporating Eringen's small-scale coefficient $e_{0}$ a into the Timoshenko beam model as defined by Wang et al.[7]:

$E I \frac{d^{2} \phi}{d x^{2}}-\kappa G A\left(\phi+\frac{d w}{d x}\right)+\rho I \omega^{2} \phi-\left(e_{0} \bar{a}\right)^{2}\left(\rho A \omega^{2} \frac{d w}{d x}+\rho I \omega^{2} \frac{d^{2} \phi}{d x^{2}}\right)=0$

$\kappa G A\left(\frac{d \phi}{d x}+\frac{d^{2} w}{d x^{2}}\right)+\rho A \omega^{2} w=0$

where $\phi$ is the rotation, $\mathrm{w}$ is the transverse displacement, $\omega$ is the circular frequency, $\kappa$ is the Timoshenko shear correction factor, $\mathrm{G}$ is the shear modulus, $\mathrm{A}$ is the cross-section of the beam, $\mathrm{I}$ is the second moment of area, $\mathrm{e}_{0}$ is a constant specific to each material and $\bar{a}$ is the internal characteristic length in the NLT beam. In equation $17, e_{0} \bar{a}$ can be normalised and represented by $\alpha$. After decoupling equations (2) and (3) and applying free-free boundary conditions, the following equation may be derived:

$\cosh \beta \cos \gamma+\left(\frac{\left[\left(H_{2} H_{3}\right)^{2}-\left(H_{1} H_{4}\right)^{2}\right]}{2 H_{1} H_{2} H_{3} H_{4}}\right) \sinh \beta \sin \gamma=1$

where:

$H_{1}=\alpha^{2} \lambda^{2}+\left(\frac{\alpha^{2} \lambda^{2}}{\xi^{2}}-1\right) \beta \Psi_{\beta}$

$H_{2}=\alpha^{2} \lambda^{2}+\left(\frac{\alpha^{2} \lambda^{2}}{\xi^{2}}-1\right) \gamma \Psi_{\gamma}$

$H_{3}=\Psi_{\beta}+\beta$

$H_{4}=\Psi_{\gamma}+\gamma$

$a=\left(1-\frac{\alpha^{2} \lambda^{2}}{\xi^{2}}\right)$

$b=\lambda^{2}\left(\Omega+-\frac{1-\Omega \alpha^{2} \lambda^{2}}{\xi^{2}}+\alpha^{2}\right)$

$c=\lambda^{2}\left(\frac{\lambda^{2} \Omega}{\xi^{2}}-1\right)$

$\left(\begin{array}{l}\beta \\ \gamma\end{array}\right)=\left(\frac{ \pm b+\sqrt{b^{2}-4 a c}}{2 a}\right)^{1 / 2}$

were $a, b$ and $c$ are the usual quadratic formula constants and

$\Psi_{\beta}=-\frac{\beta^{2}+\lambda^{2} \Omega}{\beta}$

$\Psi_{\gamma}=\frac{\gamma^{2}+\lambda^{2} \Omega}{\gamma}$

The parameters $\mathrm{H} 1$ to $\mathrm{H} 4$ are defined by C. M. Wang et al. [7] while the derivation of equation (4) is entirely new.

The non-dimensional parameters used in the above equations are:

$\lambda=\omega^{(1 / 2)}\left(\rho A L^{4} / E I\right)^{1 / 4}$

$\Omega=E I /\left(K_{s} G A L^{2}\right)$

$\alpha=e_{0} \bar{a} / L$

$\xi=L(A / I)^{1 / 2}$

in which $\lambda$ is the dimensionless frequency parameter, $\Omega$ is the shear deformation parameter, $\alpha$ is the scaling effect parameter, $\bar{a}$ is the internal characteristic length, and finally, $\xi$ is the slenderness ratio. $\bar{x}$ and $\bar{w}$ are displacements in axial, $x$, and transverse, $y$, direction normalised with respect to length, $L$.

\subsection{Comparison with ANSYS FE Results to Identify $\alpha$}

Equation (4) was solved by applying the bisection method to identify the normalised transverse modal frequencies for the first 37 modes of a beam with aspect ratio $L / d=10.4: 1$. The results are provided in figure 7 -a. By comparing the 
ANSYS finite element results and these analytical NLT results, it is possible to obtain Eringen's scale parameter for some of the heterogeneous beams models considered previously. Direct comparison between the results in figure 7-a and the finite element results given in section 2.2 suggest that it is not always possible to determine $\alpha$ for all cases considered. However, by applying a constant:

$$
\left(\lambda / \lambda_{0}\right)_{2}=\left(\lambda / \lambda_{0}\right)_{1}-\left[C_{1} \times\left(1-\left(\lambda / \lambda_{0}\right)_{\text {Mode__ } 1}\right)\right]
$$

the FE results for perforated beams with continuous boundaries could be compared with the results obtained from solving the Eringen non-local theory.

In equation (19), $\left(\lambda / \lambda_{0}\right)_{2}$ is the normalised frequency parameter of the $\mathrm{n}^{\text {th }}$ mode after shifting, $\left(\lambda / \lambda_{0}\right)_{1}$ is the normalised frequency parameter of the $\mathrm{n}^{\text {th }}$ mode from the ANSYS results and $C_{1}$ is an empirical constant for the beam type and is equal to 1.2. The value of $C_{1}$ was obtained by curve fitting and changing the $C_{1}$ value until the NLT beam results match the results obtained from ANSYS FE. Thus, by shifting the results below the line representing the homogeneous case, obtaining $\alpha$ for beams with either voids or inclusions is possible.

The values for $\alpha$ obtained via this curve fitting method for beams with continuous surfaces show that for a given volume fraction $\alpha$ is not size independent as illustrated in figure 7-b and therefore, it cannot be considered as a unique property of the material. For the samples with textured boundaries, it is not possible to identify $\alpha$ because of the inverted size effect.

\section{An Analytical Micropolar Beam Model}

\subsection{D Micropolar elastic beam model}

In classical (Cauchy) elasticity the stress-strain relations are given by:

$\tau_{i j}=\lambda^{*} \varepsilon_{k k} \delta_{i j}+2 \mu^{*} \varepsilon_{i j}$

where $\lambda^{*}$ and $\mu^{*}$ are the Lamé constants. However, in linear, three dimensional, micropolar elasticity the force stresses, $\boldsymbol{\tau}_{\boldsymbol{i j}}$, and couple stresses, $\boldsymbol{m}_{\boldsymbol{i j}}$, are related to the deformations by equations 21 and 22 as defined by Lake [17]:

$\boldsymbol{\tau}_{i j}=\lambda^{*} \varepsilon_{k k} \delta_{i j}+\left(2 \mu^{*}+\kappa^{*}\right) \varepsilon_{i j}+\kappa^{*} \boldsymbol{e}_{i j k}\left(\boldsymbol{\theta}_{\boldsymbol{k}}-\emptyset_{k}\right)$

$\boldsymbol{m}_{i j}=\boldsymbol{\alpha}^{*} \emptyset_{k}, \delta_{i j}+\boldsymbol{\beta}^{*} \emptyset_{i},+\gamma^{*} \emptyset_{j}$

where $\emptyset$ is the microrotation and $\theta$ is the conventional macro rotation. $i, j, k=1,2,3$ and $\delta$ is the Kronecker delta which is equal to 1 if $i=j$ otherwise it is zero and $e_{i j k}$ is the permutation tensor. For even permutation of $i j k, e_{i j k}=$ +1 , for the odd permutation of ijk, $e_{i j k}=-1$ and otherwise zero. $\alpha^{*}, \beta^{*}, \gamma^{*}$ and $\kappa^{*}$ are micropolar elastic constants.

Equation (23) defines the strain components, $\boldsymbol{\varepsilon}$, in terms of the displacements, $\boldsymbol{u}$, and micro-rotations, $\emptyset$, by:

$\varepsilon_{i j}=u_{j},+e_{i j k} \emptyset_{k}$

Macro rotation and strain tensors are:

$\boldsymbol{\theta}_{\boldsymbol{k}}=\left(\boldsymbol{e}_{\boldsymbol{i j k}} \boldsymbol{u}_{\boldsymbol{k}, \boldsymbol{j}}\right) / 2$

$\varepsilon_{i j}=\left(\boldsymbol{u}_{i, j}+\boldsymbol{u}_{j, i}\right) / 2$

In equations (21) and (22), there are four additional elastic constants $\alpha^{*}, \beta^{*}, \gamma^{*}$ and $\kappa^{*}$. However, in 2D Micropolar elasticity the number of constants reduces to 4 independent engineering constants defined thus:

$$
\begin{aligned}
& E_{m}=\frac{\left(2 \mu^{*}+\kappa^{*}\right)\left(3 \lambda^{*}+2 \mu^{*}+\kappa^{*}\right)}{\left(2 \lambda^{*}+2 \mu^{*}+\kappa^{*}\right)} \\
& v_{m}=\frac{\lambda^{*}}{\left(2 \lambda^{*}+2 \mu^{*}+\kappa^{*}\right)} \\
& l_{b}^{2}=\frac{\gamma^{*}}{2\left(2 \mu^{*}+\kappa^{*}\right)}
\end{aligned}
$$




$$
N^{2}=\frac{\kappa^{*}}{2\left(\mu^{*}+\kappa^{*}\right)}
$$

where $E_{m}$ is the micropolar modulus $v_{m}$ is the micropolar Poisson's ratio and $l_{b}$ is a length scale parameter termed the characteristic length in bending that should reflect the microstructural scale. $N$ is the coupling number that quantifies the shear stress asymmetry.

In the dynamic case micro inertia also needs to be included according to:

$\boldsymbol{\tau}_{\boldsymbol{i j}, \boldsymbol{i}}=\rho \ddot{u}_{j}$

$\boldsymbol{m}_{\boldsymbol{i j}, \boldsymbol{i}}+e_{j i k} \boldsymbol{\tau}_{\boldsymbol{i j}}=\rho \boldsymbol{J}_{\boldsymbol{j} \boldsymbol{i}} \ddot{\emptyset}_{\boldsymbol{k}}$,

where $\boldsymbol{J}_{j i}$ is the microinertia tensor, double dotted displacement and macro rotation with a comma sign indicate second derivatives with respect to time. The value of $0.0325 \mathrm{~mm}^{2}$ is chosen for microinertia [21].

According to equation (21) and the stiffness matrix provided by Lakes as detailed in reference [17], the shear stress and strain relationship may be written in matrix form:

$\left[\begin{array}{l}\tau_{y x} \\ \tau_{x y}\end{array}\right]=\left[\begin{array}{ll}G_{11} & G_{12} \\ G_{21} & G_{22}\end{array}\right]\left[\begin{array}{l}\varepsilon_{y x} \\ \varepsilon_{x y}\end{array}\right]$

$G_{11}=G_{22}=\mu^{*}+\kappa^{*}$

$G_{12}=G_{21}=\mu^{*}$

Thus:

$\left[\begin{array}{c}\tau_{y x} \\ \tau_{x y}\end{array}\right]=\frac{E_{m}}{\left(1+v_{m}\right)}\left[\begin{array}{ll}\frac{1}{2\left(1-N^{2}\right)} & \frac{\left(1-2 N^{2}\right)}{2\left(1-N^{2}\right)} \\ \frac{\left(1-2 N^{2}\right)}{2\left(1-N^{2}\right)} & \frac{1}{2\left(1-N^{2}\right)}\end{array}\right]\left[\begin{array}{l}\varepsilon_{y x} \\ \varepsilon_{x y}\end{array}\right]$

indicating that the asymmetric components of the shear stress are controlled by $\mathrm{N}$ according to:

$G_{s y}=\left(G_{11}+G_{12}\right)=2 \mu^{*}+\kappa^{*}=\frac{E}{1+v_{m}}$

$G_{a s y}=\left(G_{11}-G_{12}\right)=\kappa^{*}=\frac{E}{1+v_{m}}\left(\frac{N^{2}}{1-N^{2}}\right)$

this parameter can be identified from the higher order modes using an iteration method that will be described since these involve increased shear deformation.

The solid must behave in a classical homogeneous manner if $\alpha^{*}, \beta^{*}, \gamma^{*}$ and $\kappa^{*}$ equal zero, while if $\mathrm{N}=1$ and therefore, microrotation and macrorotation are not kinematically distinct, implying that they are equal, such that $\phi_{z} \cong \theta_{z}$

\subsection{Identifying the micropolar elastic constants}

\subsubsection{Characteristic length in bending, $l_{b}$}

$E_{m}$ and $v_{m}$ can be determined from static tensile tests where no size effect is anticipated while $l_{b}$ can be obtained from mode 1 dynamic behaviour as outlined here.

In a slender beam where $d / L \ll 1$

Microrotation $\emptyset_{z} \cong$ Macrorotation $\theta_{z}$

The bending moment, $M$, is related to the internal force stress, $\tau_{\mathrm{xx}}$, and couple stress, $\mathrm{m}_{\mathrm{xz}}$, thus:

$M=\int_{A}\left(y \tau_{x x}+m_{x z}\right) d A$

Also,

$\frac{1}{R}=\frac{d \theta}{d x}=\frac{d \emptyset_{z}}{d x}=-\frac{d^{2} W}{d x^{2}}$

where $\mathrm{R}$ is the radius of curvature and $\mathrm{W}$ is the transverse displacement.

The in-plane couple stress $\mathrm{m}_{\mathrm{xz}}$ and the normal stress $\tau_{\mathrm{xx}}$ are:

$m_{x z}=\gamma^{*} \frac{d \emptyset_{z}}{d x}$

$\tau_{x x}=\frac{E_{m f} y}{R}$ 
where $E_{m f}$ is the micropolar flexural modulus.

If $\emptyset_{z} \cong \theta_{z}$ then:

$m_{x z}=\frac{\gamma^{*}}{R}$

The second moment of area is defined as:

$I=\int_{A} y^{2} d A$

where $A$ is the cross-section of the slender beam:

$A=\int_{A} d A$

Substituting for $\frac{1}{R}=-\frac{d^{2} W}{d x^{2}}$ in the moment-curvature relationship:

$\frac{d^{2} W}{d x^{2}}=-\frac{M}{E_{m f} I+\gamma^{*} A}=-\frac{M}{D_{m f}}$

$D_{m f}=E_{m f} I+\gamma^{*} A$

where $D_{m f}$ is the micropolar flexural rigidity

For an unloaded Euler-Bernoulli beam in the dynamic case:

$D_{m f} \frac{d^{4} W}{d x^{4}}-\mu \omega^{2} W=0$

where $\mu$ is the mass per unit length given by:

$\mu=\rho A$

and

$\omega=\lambda^{2} \sqrt{\frac{D_{m f}}{\rho A L^{4}}} \rightarrow \omega=\lambda^{2} \sqrt{\frac{E_{m f^{I}+\gamma^{*} A}}{\rho A L^{4}}}$

Also,

$D_{m f}=D=E I \quad$ in absence of any couple stress

The equations (43) and (44) may also be written as:

$I=\frac{b d^{3}}{12}$

$A=b d$

By combining the equations (26) and (28):

$\gamma^{*}=\frac{\boldsymbol{E}_{\boldsymbol{m} \boldsymbol{f}} l_{c}^{2}}{12}$

Note that parameter $l_{c}$ [18] differs from the conventional characteristic length in bending, $l_{b}$, by a factor of 24 .

By substituting equations (50), (51) and (52) into equation (49):

$\omega^{2}=\frac{E_{m f} \lambda^{4}}{12 \rho L^{4}}\left(d^{2}+l_{c}^{2}\right)$

Rearrange the equation (53) regarding mass and frequency:

$m . \omega^{2}=\frac{E_{m f} \lambda^{4} b}{12}\left(\frac{d}{L}\right)^{3}\left(1+\left(\frac{l_{c}}{d}\right)^{2}\right)$

Equation (54) thus relates the characteristic length, $l_{c}$, non-dimensional frequency, $\lambda$, micropolar flexural modulus, $\mathrm{E}_{\mathrm{mf}}$, and beam dimensions, to the product of beam's mass, $\mathrm{m}$ ( $\mathrm{m}$ without subscript is scalar and stands for mass), 
multiplied by squared frequency. Thus, if this product is determined for beams of various size and plotted against the beam's reciprocal size measure, $\left(1 / \mathrm{d}^{2}\right)$, then it is possible to obtain $\mathrm{E}_{\mathrm{mf}}$ or $\lambda$ from the intercept and the characteristic length from the slope. Since equation (54) assumes the beam is slender, it is only applied to mode one here where this assumption is presumed valid.

Equation (54) was used to obtain the characteristic length of bending for the following beam types:

1) Perforated beams with continuous boundaries

2) Beams with compliant inclusions and continuous boundaries

3) Beams with compliant matrix and textured boundaries

Only the characteristic length for the above beam types are listed in table 3 , and these values were subsequently used to estimate the coupling number in section 6 . Other beam types did not satisfy the micropolar theory.

The characteristic length does not vary with beam size and only depends on volume fraction. See table 3 below:

Table 3: Characteristic length changes with volume fraction

\begin{tabular}{|c|c|c|c|}
\hline \multirow{2}{*}{$\begin{array}{c}\text { Void or } \\
\text { inclusion vol. } \\
\text { fraction }\end{array}$} & \multicolumn{2}{|c|}{$\mathrm{I}_{\mathrm{c}}$ for beams with continuous boundaries, $\mathrm{mm}$} & \multirow{2}{*}{$\begin{array}{l}\mathrm{I}_{\mathrm{c}} \text { for beams with textured } \\
\text { boundaries, } \mathrm{mm}\end{array}$} \\
\hline & Perforated beams & Beams with compliant inclusions & \\
\hline $4 \%$ & 0.2717 & 0.2555 & 0.2218 \\
\hline $8 \%$ & 0.4139 & 0.3612 & 0.3126 \\
\hline $15 \%$ & 0.5432 & 0.4681 & 0.3829 \\
\hline $23 \%$ & 0.6522 & 0.5621 & 0.4379 \\
\hline $33 \%$ & 0.7334 & 0.6367 & 0.4759 \\
\hline
\end{tabular}

\subsubsection{Obtaining the coupling number}

Equation (54) only facilitates the identification of the characteristic length parameter from the first flexural natural frequency based on the assumption of slender beam behaviour for which shear deformation is negligible.

However, now that the characteristic length has been determined, the coupling number, $N$, may be estimated from the ANSYS finite element results for the higher flexural frequencies using a numerical control volume finite element method (CVFEM) which has been specifically developed in Matlab and incorporates micropolar theory. The CVFEM is capable of modelling, meshing and performing both static deformation and dynamic modal analysis. The CVFEM is used in combination with an iterative method to match or fit its predictions to the ANSYS results.

The iteration process is based on linear regression as described by Beveridge et al. [18] and fits the curves in the graphs for $m . \omega^{2}$ vs mode numbers (or wavelength). The first two transverse modes are used in the process to iterate for coupling number, $\mathrm{N}$, which, as a constitutive property, should satisfy all modal frequencies and all model depths. A second Matlab code is also developed which automatically estimates $\mathrm{N}$ by linear regression. The remainder of this paper describes the development and utilization of the CVFEM code.

\section{Development of the Numerical CVFEM Code Incorporating Micropolar Elasticity}

\subsection{Determination of Mass and Microinertia Matrices}

Since an exact solution for the transverse vibration of a 2D micropolar beam is not available, a 2D numerical method based on the CVFEM was developed. The Matlab CVFEM code incorporates a dual mesh of control volumes over a finite element mesh. Thus control volumes are constructed around each node of six-node triangular finite elements. Discrete equilibrium equations are developed for each control volume by integrating the stress resultants around each control volume face to yield the stiffness matrix. The method was originally developed for predicting static deformations by Beveridge et al.[9], [18]. This work exploits the method used to derive the stiffness matrix since it is already validated for the static case by Beverage et al. However, the method has now been significantly enhanced through the incorporation of mass and microinertia matrices to facilitate the dynamic analysis of micropolar media.

According to micropolar theory, each node can move in $\mathrm{x}$ and $\mathrm{y}$ directions and rotate about its centre. 
A consistent mass matrix was obtained for the 6-node triangular element using equation (55):

$M_{C}=\int_{\Omega} \rho(\mathbb{N})^{T} \mathbb{N} d \Omega$

$\mathbb{N}$ is the shape function, and T stands for transposed.

$M_{c}$ is the consistent mass matrix and is an integration of mass over the domain $\Omega$ which is the area of a triangular element within the mesh, figure 8.

A lumped mass matrix $M_{L}$ was also defined,

$M_{L}=\frac{\rho A}{6} \operatorname{diag}\left[\begin{array}{lllll}1 & 1 & 1 & \ldots & 1\end{array}\right]^{18 \times 18}$

then the template mass matrix obtained for each element within the mesh using the equation below:

$M_{T}=\delta M_{L}+(1-\delta) M_{C}$

$\delta$ is a scaling coefficient and set at 0.5 to count for both lumped and consistent mass matrix properties. Details of this method is discussed by Felippa et al [22].

In equation (31), the microinertia tensor, $\boldsymbol{J}_{\boldsymbol{j} i}$ is considered as equal in all directions for simplicity. Therefore, in order to include microinertia into the formulation, it must be assembled into the elements of an overall mass matrix that are associated with the micro rotation. Thus, the microinertia matrix is incorporated into the overall mass matrix.

Having obtained stiffness and mass matrices for an element, assembly of global mass and stiffness matrixes can then be carried out following standard finite element procedures. Finally, the eigenvalue equation below can be solved in the absence of external loads:

$K-\omega^{2} M=0$

to obtain the natural frequencies. Here $\mathrm{K}$ and $\mathrm{M}$ are the global stiffness and overall mass matrices respectively.

By solving the eigenvalue problem, equation (58), $\omega^{2}$ will be an array comprised of the diagonal elements of the resulting matrix. The square root of the array elements provides frequency spectrum. Once the modal frequencies are obtained the normalised displacements components can be extracted, and the mode shapes obtained.

\subsection{CVFEM Modelling of Beam Samples}

Straight sided and equal sized triangular elements were used for modelling all four sizes of beam. The CVFEM mesh comprised of 4 and 20 element divisions through the depth and along the length respectively to give a model with 1107 degrees of freedom in total. Overall dimensions of each model correspond to the beam sizes used in ANSYS finite element models. Figure 8 shows the mesh assembled from triangular elements by the CVFEM analysis. It is important to note that since the CVFEM code incorporates micropolar rather than classical constitutive behaviour, it is capable of automatically modelling and meshing the desired beams without having to incorporate the geometric details of the voids or inclusions as was necessary in the ANSYS models.

\section{CVFEM Results and Discussion}

\subsection{Approximation of the Coupling number, $\mathrm{N}$}

\subsubsection{Estimation of $N$ for beams with continuous boundaries}

Using the CVFEM code and linear regression as described in section 4.2.2, unique values for $\mathrm{N}$ were identified. In figures 9-a and 9-b, the ANSYS finite element results and the results predicted for mode one and two by the micropolar CVFEM code are compared after convergence of the iteration process with $m \omega^{2}$ being obtained within the range of $\mathrm{N}$ from 0 to 0.9 . The first value represents the lower bound on $\mathrm{N}$ and corresponds to the classical case while the second value is an approximation to the upper bound on $\mathrm{N}$ of 1 corresponding to the constrained micropolar or so called couple stress elasticity case. Note that setting the upper bound for $\mathrm{N}$ at 1 would give rise to numerical error.

The linear regression method was applied to the first and second modal frequencies (obtained from ANSYS finite element analysis) for all beam sizes and a void or inclusion volume fraction of 0.23 . In theory, the more mode numbers used for iteration, the more accurate the coupling number estimate will be, but as the frequency spectrum identified by the CVFEM also includes longitudinal modes above mode 2, only the first two modal frequencies were used in the iteration process. The unique value of $\mathrm{N}$ identified in this way was 0.054 for beams with voids and 0.053 for beams with compliant inclusions. 
The iteration process was similarly performed for all other inclusion volume fractions from $4 \%$ to $33 \%$. This revealed that $\mathrm{N}$ changes only slightly with volume fraction, figure 10.

As a further verification, figure 11 shows the full-size effect across the first 8 modes forecast by the CVFEM using various values on $\mathrm{N}$ for the second size of the beam with continuous boundaries and voids. The value of $\mathrm{N}$ identified by the iterative procedure evidently provides the best forecast of the ANSYS results across this frequency range.

As with the characteristic length, when the boundary of the beam is intercepted by voids and inclusions, the coupling number could also not be identified by the iterative method because the values of $m \omega^{2}$ obtained from the ANSYS results exhibit a size effect that is not anticipated by the micropolar theory. This also applies to the case where the matrix material, though continuous along the boundaries, is more compliant than the inclusions.

\subsubsection{Estimation of $N$ for beams with compliant matrix and textured boundaries}

While the size effect in beams with compliant matrix and continuous surfaces contradicts the micropolar theory, when the boundaries are textured by intersection with the stiffer inclusions the size effect is as expected. Thus for this type of beam, a value for the coupling number can also be identified by the linear regression based iterative procedure. Interestingly, a unique value of 0.05 is estimated in this case which is comparable to that obtained in the previous cases considered.

\subsection{Overall Comparison of CVFEM Predictions and ANSYS FE Results}

Having quantified the characteristic length parameter using the analytical solution, equation (54), and the coupling number numerically, the CVFEM procedure was used to predict the frequency spectrum for all sizes of beams with inclusions and/or perforated by voids at all volume fractions considered. The frequency parameters $(\lambda)$ are provided in Appendix table 7 and only include three modal frequencies parameters for three types of beams: beams with voids and continuous boundaries, beams with inclusions and continuous boundaries, and finally beams with compliant matrix and textured boundaries. It was realised that the normalised frequencies $(\Lambda)$ approached one quickly and only were pronounced for the first thee modal frequencies, see figure 12 for the micropolar beams corresponding to the beams with voids and height of two unit-cells (Second smallest beam sample). When the CVFEM predictions are compared to the results obtained from ANSYS finite element analysis, figure 3, a number of similarities and differences are evident. The CVFEM results indicate that in the micropolar beam, the modal frequencies disperse quickly and that means after the first few modes the size effect is rapidly suppressed which limits the utility of the higher modes in the investigation of any size effect. On the other hand, the ANSYS results indicate that the size effect remains more pronounced and may even change in nature. Furthermore, while the CVFEM forecasts are qualitatively similar to the FE results for the low-frequency flexural modes there is some more quantitative discrepancy as seen when figure 13, which illustrates the predictions for the first mode, is compared with figure 4 . The size effect predicted is decidedly more nonlinear than that observed in the detailed FE model results.

Comparable similarities and differences are seen in the cases where the beams contain compliant inclusions and where inclusions, although more rigid, intersect the boundaries, appendix table 7.

\section{Summary and Conclusions}

ANSYS FE was used for creating beam models and generating modal frequencies and using them as the basis for comparison. This approach is different from the usual techniques in the literature but has some benefits. First of all, the analytical methods in the literature were not quite applicable to our beam models. Secondly, changing the volume fraction in beam models with a periodic array of voids or inclusions required changing material constants such as modulus of elasticity in order to keep the overall dimensions and the properties of the homogenized equivalent beam unchanged. This was possible when performing tensile test using ANSYS finite element while keeping the cell's mesh configurations constant.

Geometrically similar beam samples of different sizes that contained periodic heterogeneities were modelled using ANSYS, and modal analyses were performed to identify the unconstrained flexural natural frequencies. Contrary to the homogeneous case where these frequencies are size independent, the ANSYS results indicate that in the 
heterogeneous case they are size dependent. Moreover, this size effect depends on both the specification of the periodic heterogeneity and its location relative to the sample boundaries. In some cases, the size effect appears to be consistent with more generalised continuum descriptions of dynamic constitutive behaviour such as micropolar elasticity in that an increase in frequency with reducing size is observed while in other cases the effect is contradictory.

For cases where the ANSYS results exhibit a consistent size effect, Eringen's Nonlocal Timoshenko beam (NLT) analysis was considered in attempting to explain the dynamic behaviour observed. However, the NLT appears to have shortcomings since the small-scale coefficient was not constant for all model sizes with equal aspect ratio. The size dependency of the coefficient value thus implies that it cannot be interpreted as an independent constitutive property.

An analytical solution for the unconstrained transverse vibration of a slender micropolar beam was then derived and compared to the finite element results. This enabled the characteristic length parameter to be identified from the size dependency of the first natural frequency. However, this approach did not permit the identification of the second additional constitutive parameter, the coupling number, and therefore a numerical application of Eringen's micropolar theory in transverse vibration was investigated by developing a CVFEM code for modal analysis which can model, mesh and perform both static and modal analysis using micropolar theory to account for microrotation and couple stress. The coupling number was thus identified by an iterative approach based upon matching multiple flexural frequency forecasts provided by the code to the ANSYS results. The coupling number values obtained are towards the lower bound of the anticipated range and thus show some agreement with those obtained previously for similar materials when undergoing static deformation. Nevertheless, when these values were subsequently employed within the CVFEM code to predict the full range of frequencies covered by the FE results discernable differences were still present with these becoming more marked as frequency increased. Thus while micropolar theory appears to have some capacity to forecast the size effect at low frequencies, its predictive ability becomes progressively compromised as frequency increases for the particular heterogeneous materials considered.

\section{Appendix}

The numerical results provided in this appendix provide the transverse modal frequency parameters $(\lambda)$. The results include ANSYS finite element results, table 4 to 6 , and CVFEM results in table 7 for the beams with aspect ratio of 10.4:1

Table 4 : ANSYS results for the first ten non-dimensional bending modal frequencies, $\lambda$, of four beam sizes for beams with voids and continuous boundaries.

\begin{tabular}{|c|c|c|c|c|c|c|c|c|c|c|c|}
\hline $\mathrm{Vr} / \mathrm{Sr}$ & $1 / d^{2}$ & Mode 1 & Mode 2 & Mode 3 & Mode 4 & Mode 5 & Mode 6 & Mode 7 & Mode 8 & Mode 9 & Mode 10 \\
\hline \multirow[t]{4}{*}{0.12} & 1.33 & 4.76955 & 7.68132 & 10.35591 & 12.76693 & 14.93788 & 16.88913 & 18.61605 & 19.99604 & 22.62761 & 23.77287 \\
\hline & 0.33 & 4.68729 & 7.57493 & 10.25310 & 12.69255 & 14.91404 & 16.94335 & 18.80583 & 20.52301 & 22.11161 & 23.58229 \\
\hline & 0.15 & 4.66959 & 7.54856 & .22110 & 2.65790 & 4.87917 & 16.91035 & 18.77657 & 20.49923 & 22.09501 & 23.57460 \\
\hline & 0.08 & 4.66333 & 7.53907 & 10.20920 & 12.64431 & 14.86449 & 16.89507 & 18.76115 & 20.48404 & 22.08054 & 23.56148 \\
\hline \multirow[t]{4}{*}{0.17} & 1.33 & 4.90166 & 7.84884 & 10.51240 & 12.87028 & 14.94866 & 16.75620 & 18.25124 & 19.27956 & 23.33800 & 24.05576 \\
\hline & 0.33 & 4.72757 & 7.63200 & 10.31759 & 12.75624 & 14.97082 & 16.98881 & 18.83670 & 20.53666 & 22.10554 & 23.55362 \\
\hline & 0.15 & 4.68756 & 7.57440 & 10.25109 & 12.68880 & 14.90873 & 16.93700 & 18.79930 & 20.51731 & 22.10779 & 23.58129 \\
\hline & 0.08 & 4.67318 & 7.55331 & 10.22584 & 12.66164 & 14.88138 & 16.91078 & 18.77521 & 20.49630 & 22.09079 & 23.56949 \\
\hline \multirow[t]{4}{*}{0.23} & 1.33 & 5.06288 & 8.02794 & 10.63599 & 12.87575 & 14.78071 & 16.35164 & 17.54079 & 18.26429 & 22.58100 & 22.85800 \\
\hline & 0.33 & 4.78076 & 7.70206 & 10.38746 & 12.81145 & 15.00121 & 16.98756 & 18.79922 & 20.45959 & 21.98596 & 23.38806 \\
\hline & 0.15 & 4.71149 & 7.60675 & 10.28491 & 12.71812 & 14.92935 & 16.94617 & 18.79525 & 20.49900 & 22.07450 & 23.53231 \\
\hline & 0.08 & 4.68620 & 7.57108 & 10.24467 & 12.67837 & 14.89384 & 16.91758 & 18.77564 & 20.48990 & 22.07746 & 23.54905 \\
\hline \multirow[t]{3}{*}{0.29} & 1.33 & 5.22486 & 8.15349 & 10.61435 & 12.65910 & 14.31213 & 15.59849 & 16.50500 & 17.02099 & 20.76968 & 21.14741 \\
\hline & 0.33 & 4.83872 & 7.76881 & 10.43623 & 12.82049 & 14.95620 & 16.87921 & 18.62170 & 20.20869 & 21.65796 & 22.97855 \\
\hline & 0.15 & 4.73769 & 7.63848 & 10.31101 & 12.72910 & 14.91850 & 16.90907 & 18.72915 & 20.40219 & 21.94600 & 23.37150 \\
\hline
\end{tabular}




\begin{tabular}{|c|c|c|c|c|c|c|c|c|c|c|c|}
\hline \multirow{3}{*}{0.35} & 0.08 & 4.70036 & 7.58842 & 10.25926 & 12.68512 & 14.88911 & 16.89905 & 18.74183 & 20.44008 & 22.01115 & 23.46609 \\
\cline { 2 - 11 } & 1.33 & 5.35391 & 8.14392 & 10.37197 & 12.11473 & 13.47552 & 14.48776 & 15.17417 & 15.55343 & 18.77221 & 19.31754 \\
\cline { 2 - 11 } & 0.33 & 4.89192 & 7.80981 & 10.42461 & 12.72609 & 14.76084 & 16.57268 & 18.19838 & 19.66493 & 20.99083 & 22.18467 \\
\cline { 2 - 11 } & 0.15 & 4.76152 & 7.65909 & 10.31068 & 12.69310 & 14.83683 & 16.77533 & 18.53948 & 20.15453 & 21.63943 & 23.00600 \\
\cline { 2 - 10 } & 0.08 & 4.71287 & 7.59915 & 10.25851 & 12.66461 & 14.84301 & 16.82350 & 18.63439 & 20.29934 & 21.83652 & 23.25743 \\
\hline
\end{tabular}

Table 5: ANSYS results for the first ten non-dimensional bending modal frequencies, $\lambda$, of four beam sizes for beams with compliant inclusions and continuous boundaries.

\begin{tabular}{|c|c|c|c|c|c|c|c|c|c|c|c|}
\hline $\mathrm{Vr} / \mathrm{Sr}$ & $1 / d^{2}$ & Mode 1 & Mode 2 & Mode 3 & Mode 4 & Mode 5 & Mode 6 & Mode 7 & Mode 8 & Mode 9 & Mode 10 \\
\hline \multirow[t]{4}{*}{0.12} & 1.33 & 4.75123 & 7.66769 & 10.35911 & 12.79741 & 15.00668 & 17.01499 & 18.84630 & 20.49177 & 22.19597 & 23.59273 \\
\hline & 0.33 & 4.68110 & 7.56720 & 10.24600 & 12.68789 & 14.91294 & 16.94646 & 18.81348 & 20.53531 & 22.12848 & 23.60351 \\
\hline & 0.15 & 4.66748 & 7.54630 & 10.21966 & 12.65800 & 14.88127 & 16.91468 & 18.78318 & 20.50804 & 22.10581 & 23.58711 \\
\hline & 0.08 & 4.66249 & 7.53830 & 10.20903 & 12.64515 & 14.86659 & 16.89853 & 18.76588 & 20.49005 & 22.08768 & 23.56959 \\
\hline \multirow[t]{4}{*}{0.17} & 1.33 & 4.84870 & 7.79438 & 10.48399 & 12.89362 & 15.05104 & 16.97843 & 18.66707 & 19.97418 & 22.70293 & 23.81555 \\
\hline & 0.33 & 4.71030 & 7.60966 & 10.29603 & 12.74053 & 14.96462 & 16.99474 & 18.85674 & 20.57241 & 22.15847 & 23.62528 \\
\hline & 0.15 & 4.68058 & 7.56559 & 10.24292 & 12.68337 & 14.90744 & 16.94082 & 18.80887 & 20.53297 & 22.12978 & 23.60976 \\
\hline & 0.08 & 4.66978 & 7.54912 & 10.22220 & 12.65969 & 14.88186 & 16.91418 & 18.78177 & 20.50613 & 22.10393 & 23.58594 \\
\hline \multirow[t]{4}{*}{0.23} & 1.33 & 4.96849 & 7.94033 & 10.61103 & 12.96234 & 15.02517 & 16.80993 & 18.27300 & 19.25911 & 23.40805 & 23.92484 \\
\hline & 0.33 & 4.74841 & 7.66251 & 10.35388 & 12.79499 & 15.00981 & 17.02668 & 18.87283 & 20.57082 & 22.13765 & 23.58338 \\
\hline & 0.15 & 4.69778 & 7.58987 & 10.27030 & 12.71048 & 14.93192 & 16.96124 & 18.82433 & 20.54298 & 22.13400 & 23.60783 \\
\hline & 0.08 & 4.67937 & 7.56279 & 10.23785 & 12.67553 & 14.89668 & 16.92727 & 18.79279 & 20.51494 & 22.11055 & 23.59024 \\
\hline \multirow[t]{4}{*}{0.29} & 1.33 & 5.09188 & 8.07596 & 10.70363 & 12.96512 & 14.89632 & 16.50033 & 17.72840 & 18.48490 & 22.82080 & 23.10764 \\
\hline & 0.33 & 4.78987 & 7.71682 & 10.40761 & 12.83679 & 15.03172 & 17.02332 & 18.84050 & 20.50669 & 22.03927 & 23.44780 \\
\hline & 0.15 & 4.71681 & 7.61537 & 10.29664 & 12.73282 & 14.94694 & 16.96665 & 18.81872 & 20.52549 & 22.10404 & 23.56488 \\
\hline & 0.08 & 4.69009 & 7.57735 & 10.25318 & 12.68902 & 14.90650 & 16.93228 & 18.79233 & 20.50866 & 22.09828 & 23.57190 \\
\hline \multirow[t]{4}{*}{0.35} & 1.33 & 5.19900 & 8.18027 & 10.75120 & 12.91266 & 14.70869 & 16.14526 & 17.19202 & 17.80838 & 21.86749 & 22.21757 \\
\hline & 0.33 & 4.82774 & 7.76310 & 10.44718 & 12.85702 & 15.02368 & 16.98069 & 18.75855 & 20.38153 & 21.86718 & 23.22472 \\
\hline & 0.15 & 4.73465 & 7.63768 & 10.31667 & 12.74478 & 14.94674 & 16.95144 & 18.78654 & 20.47502 & 22.03434 & 23.47507 \\
\hline & 0.08 & 4.70048 & 7.59047 & 10.26515 & 12.69652 & 14.90715 & 16.92448 & 18.77513 & 20.48139 & 22.06051 & 23.52323 \\
\hline
\end{tabular}

Table 6: ANSYS results for the first ten non-dimensional bending modal frequencies, $\lambda$, of four beam sizes with compliant matrix and textured boundaries

\begin{tabular}{|c|c|c|c|c|c|c|c|c|c|c|c|}
\hline $\mathrm{Vr} / \mathrm{Sr}$ & $1 / \mathrm{d}^{2}$ & Mode 1 & Mode 2 & Mode 3 & Mode 4 & Mode 5 & Mode 6 & Mode 7 & Mode 8 & Mode 9 & Mode 10 \\
\hline \multirow{3}{*}{0.12} & 1.33 & 4.72946 & 7.63167 & 10.31391 & 12.74914 & 14.96101 & 16.97705 & 18.82349 & 20.52080 & 22.08456 & 23.51864 \\
\cline { 2 - 11 } & 0.33 & 4.67474 & 7.55630 & 10.23092 & 12.66928 & 14.89158 & 16.92312 & 18.78891 & 20.51027 & 22.10379 & 23.58024 \\
\cline { 2 - 12 } & 0.15 & 4.66415 & 7.54046 & 10.21133 & 12.64735 & 14.86851 & 16.90006 & 18.76707 & 20.49073 & 22.08789 & 23.56936 \\
\cline { 2 - 11 } & 0.08 & 4.66041 & 7.53475 & 10.20404 & 12.63884 & 14.85907 & 16.88991 & 18.75639 & 20.47983 & 22.07692 & 23.55855 \\
\hline 0.17 & 1.33 & 4.79981 & 7.73649 & 10.44016 & 12.88522 & 15.09868 & 17.11120 & 18.95153 & 20.64153 & 22.19873 & 23.62610 \\
\cline { 2 - 12 } & 0.33 & 4.69361 & 7.58611 & 10.26980 & 12.71521 & 14.94282 & 16.97809 & 18.84626 & 20.56862 & 22.16152 & 23.63539 \\
\cline { 2 - 11 } & 0.15 & 4.67256 & 7.55394 & 10.22929 & 12.66920 & 14.89381 & 16.92850 & 18.79839 & 20.52477 & 22.12430 & 23.60763 \\
\cline { 2 - 11 } & 0.08 & 4.66512 & 7.54233 & 10.21420 & 12.65134 & 14.87374 & 16.90666 & 18.77518 & 20.50069 & 22.09963 & 23.58306 \\
\hline \multirow{2}{*}{0.23} & 1.33 & 4.86778 & 7.83361 & 10.54994 & 12.99331 & 15.19525 & 17.19013 & 19.00983 & 20.67820 & 22.21484 & 23.62362 \\
\cline { 2 - 11 } & 0.33 & 4.71272 & 7.61511 & 10.30545 & 12.75433 & 14.98271 & 17.01645 & 18.88109 & 20.59805 & 22.18360 & 23.64764 \\
\cline { 2 - 11 } & 0.15 & 4.68105 & 7.56693 & 10.24559 & 12.68764 & 14.91352 & 16.94881 & 18.81879 & 20.54492 & 22.14375 & 23.62573 \\
\cline { 2 - 10 } & 0.08 & 4.66980 & 7.54951 & 10.22324 & 12.66163 & 14.88488 & 16.91839 & 18.78735 & 20.51312 & 22.11233 & 23.59574 \\
\hline
\end{tabular}




\begin{tabular}{|c|c|c|c|c|c|c|c|c|c|c|c|}
\hline 0.29 & 1.33 & 4.92902 & 7.91767 & 10.63896 & 13.07209 & 15.25356 & 17.22171 & 19.01125 & 20.64896 & 22.15520 & 23.53770 \\
\cline { 2 - 11 } & 0.33 & 4.73121 & 7.64217 & 10.33705 & 12.78652 & 15.01227 & 17.04078 & 18.89797 & 20.60541 & 22.17930 & 23.62915 \\
\cline { 2 - 11 } & 0.15 & 4.68972 & 7.57979 & 10.26091 & 12.70387 & 14.92945 & 16.96357 & 18.83174 & 20.55554 & 22.15156 & 23.63006 \\
\cline { 2 - 11 } & 0.08 & 4.67484 & 7.55698 & 10.23220 & 12.67124 & 14.89453 & 16.92768 & 18.79602 & 20.52104 & 22.11932 & 23.60152 \\
\hline 0.35 & 1.33 & 4.97450 & 7.97691 & 10.69532 & 13.11107 & 15.26453 & 17.19681 & 18.94450 & 20.53606 & 21.99261 & 23.32542 \\
\cline { 2 - 11 } & 0.33 & 4.74526 & 7.66210 & 10.35901 & 12.80674 & 15.02757 & 17.04848 & 18.89564 & 20.59065 & 22.14966 & 23.58161 \\
\cline { 2 - 11 } & 0.15 & 4.69628 & 7.58920 & 10.27154 & 12.71418 & 14.93820 & 16.96979 & 18.83470 & 20.55444 & 22.14572 & 23.61878 \\
\cline { 2 - 11 } & 0.08 & 4.67845 & 7.56215 & 10.23804 & 12.67693 & 14.89946 & 16.93139 & 18.79821 & 20.52143 & 22.11778 & 23.59761 \\
\hline
\end{tabular}

Table 7: CVFEM results for the non-dimensional modal frequencies $(\lambda)$ of modes one to three of models with various beam depth and void or inclusions radius.

\begin{tabular}{|c|c|c|c|c|c|c|c|c|c|c|}
\hline \multirow[b]{2}{*}{$\mathrm{Vr} / \mathrm{Sr}$} & \multirow[b]{2}{*}{$1 / d^{\wedge} 2$} & \multicolumn{3}{|c|}{$\begin{array}{c}\text { Beam with voids and continuous } \\
\text { boundaries }\end{array}$} & \multicolumn{3}{|c|}{$\begin{array}{l}\text { Beam with compliant inclusions and } \\
\text { continuous boundaries }\end{array}$} & \multicolumn{3}{|c|}{$\begin{array}{c}\text { Beam with compliant matrix and } \\
\text { continuous boundaries }\end{array}$} \\
\hline & & Mode 1 & Mode 2 & Mode 3 & Mode 1 & Mode 2 & Mode 3 & Mode 1 & Mode 2 & Mode 3 \\
\hline \multirow[t]{4}{*}{0.12} & 1.33 & 5.55048 & 7.93926 & 10.48025 & 5.49408 & 7.91719 & 10.46336 & 5.38661 & 7.86623 & 10.42483 \\
\hline & 0.33 & 5.19436 & 7.88419 & 10.43642 & 5.14068 & 7.85885 & 10.41906 & 5.05858 & 7.81050 & 10.38362 \\
\hline & 0.15 & 4.98113 & 7.80541 & 10.39748 & 4.94219 & 7.78019 & 10.38063 & 4.88770 & 7.73798 & 10.34900 \\
\hline & 0.08 & 4.86615 & 7.73972 & 10.36377 & 4.83879 & 7.71720 & 10.34784 & 4.80220 & 7.68259 & 10.32010 \\
\hline \multirow[t]{4}{*}{0.17} & 1.33 & 5.68121 & 7.95820 & 10.49617 & 5.61747 & 7.93569 & 10.47906 & 5.50346 & 7.88422 & 10.44059 \\
\hline & 0.33 & 5.42876 & 7.94097 & 10.46926 & 5.34149 & 7.91189 & 10.44894 & 5.23072 & 7.85958 & 10.41067 \\
\hline & 0.15 & 5.20517 & 7.89103 & 10.44088 & 5.12100 & 7.85607 & 10.41894 & 5.03132 & 7.80451 & 10.38242 \\
\hline & 0.08 & 5.04767 & 7.83712 & 10.41432 & 4.97748 & 7.79957 & 10.39155 & 4.90944 & 7.75156 & 10.35719 \\
\hline \multirow[t]{4}{*}{0.23} & 1.33 & 5.73075 & 7.96448 & 10.50144 & 5.67330 & 7.94297 & 10.48519 & 5.55134 & 7.89058 & 10.44605 \\
\hline & 0.33 & 5.55249 & 7.96269 & 10.48345 & 5.46755 & 7.93613 & 10.46412 & 5.32718 & 7.87986 & 10.42287 \\
\hline & 0.15 & 5.35917 & 7.93005 & 10.46195 & 5.26393 & 7.89744 & 10.44042 & 5.13061 & 7.83717 & 10.39878 \\
\hline & 0.08 & 5.19684 & 7.88978 & 10.44050 & 5.10667 & 7.85243 & 10.41761 & 4.99336 & 7.79093 & 10.37650 \\
\hline \multirow[t]{4}{*}{0.29} & 1.33 & 5.75314 & 7.96717 & 10.50370 & 5.70056 & 7.94631 & 10.48799 & 5.57569 & 7.89363 & 10.44864 \\
\hline & 0.33 & 5.61768 & 7.97255 & 10.49037 & 5.54080 & 7.94800 & 10.47215 & 5.38462 & 7.89020 & 10.42948 \\
\hline & 0.15 & 5.45414 & 7.94918 & 10.47319 & 5.36179 & 7.91960 & 10.45279 & 5.19809 & 7.85527 & 10.40823 \\
\hline & 0.08 & 5.30125 & 7.91787 & 10.45513 & 5.20666 & 7.88355 & 10.43323 & 5.05576 & 7.81461 & 10.38796 \\
\hline \multirow[t]{4}{*}{0.35} & 1.33 & 5.76408 & 7.96846 & 10.50478 & 5.71468 & 7.94799 & 10.48940 & 5.58828 & 7.89516 & 10.44993 \\
\hline & 0.33 & 5.65203 & 7.97737 & 10.49389 & 5.58251 & 7.95417 & 10.47651 & 5.41700 & 7.89556 & 10.43304 \\
\hline & 0.15 & 5.50881 & 7.95888 & 10.47925 & 5.42348 & 7.93165 & 10.45993 & 5.23936 & 7.86506 & 10.41353 \\
\hline & 0.08 & 5.36639 & 7.93277 & 10.46330 & 5.27535 & 7.90141 & 10.44256 & 5.09632 & 7.82805 & 10.39453 \\
\hline
\end{tabular}


Table 8: Correction of modulus of elasticity of beams matrix by void or inclusions radius for various beam models

\begin{tabular}{|c|c|c|c|c|c|c|}
\hline \multirow{2}{*}{$\begin{array}{c}\text { Void or inclusion } \\
\text { radius, } \mathrm{mm}\end{array}$} & \multicolumn{5}{|c|}{\begin{tabular}{c} 
The modulus of elasticity of matrix, MPa \\
\cline { 2 - 7 }
\end{tabular}} & \multicolumn{2}{|c|}{ Perforated beams } & \multicolumn{2}{c|}{$\begin{array}{c}\text { Beams with compliant } \\
\text { inclusions }\end{array}$} & \multicolumn{2}{c|}{ Beams with compliant matrix } \\
\cline { 2 - 7 } & $\begin{array}{c}\text { Continuous } \\
\text { boundaries }\end{array}$ & $\begin{array}{c}\text { Textured } \\
\text { boundaries }\end{array}$ & $\begin{array}{c}\text { Continuous } \\
\text { boundaries }\end{array}$ & $\begin{array}{c}\text { Textured } \\
\text { boundaries }\end{array}$ & $\begin{array}{c}\text { Continuous } \\
\text { boundaries }\end{array}$ & $\begin{array}{c}\text { Textured } \\
\text { boundaries }\end{array}$ \\
\hline 0 & $7.000 \mathrm{E}+04$ & $7.000 \mathrm{E}+04$ & $7.000 \mathrm{E}+04$ & $7.000 \mathrm{E}+04$ & $7.000 \mathrm{E}+04$ & $7.000 \mathrm{E}+04$ \\
\hline 0.1 & $7.796 \mathrm{E}+04$ & $7.814 \mathrm{E}+04$ & $7.590 \mathrm{E}+04$ & $7.600 \mathrm{E}+04$ & $6.679 \mathrm{E}+04$ & $6.681 \mathrm{E}+04$ \\
\hline 0.15 & $8.892 \mathrm{E}+04$ & $8.915 \mathrm{E}+04$ & $8.382 \mathrm{E}+04$ & $8.394 \mathrm{E}+04$ & $6.292 \mathrm{E}+04$ & $6.295 \mathrm{E}+04$ \\
\hline 0.2 & $1.064 \mathrm{E}+05$ & $1.063 \mathrm{E}+05$ & $9.600 \mathrm{E}+04$ & $9.599 \mathrm{E}+04$ & $5.775 \mathrm{E}+04$ & $5.776 \mathrm{E}+04$ \\
\hline 0.25 & $1.338 \mathrm{E}+05$ & $1.328 \mathrm{E}+05$ & $1.139 \mathrm{E}+05$ & $1.135 \mathrm{E}+05$ & $5.142 \mathrm{E}+04$ & $5.142 \mathrm{E}+04$ \\
\hline 0.3 & $1.796 \mathrm{E}+05$ & $1.767 \mathrm{E}+05$ & $1.404 \mathrm{E}+05$ & $1.397 \mathrm{E}+05$ & $4.412 \mathrm{E}+04$ & $4.413 \mathrm{E}+04$ \\
\hline
\end{tabular}

Table 9: Correction of density by void or inclusions radius

\begin{tabular}{|c|c|c|c|}
\hline \multirow{2}{*}{$\begin{array}{c}\text { Void or inclusion } \\
\text { radius, } \mathrm{mm}\end{array}$} & \multicolumn{3}{|c|}{ The mass density of matrix and inclusions, $\mathrm{kg} / \mathrm{m}^{3}$} \\
\cline { 2 - 4 } & Perforated beams & Beams with inclusions \\
\cline { 2 - 4 } & For matrix & For matrix & For inclusions \\
\hline 0 & 2700.00 & 2700.00 & N/A \\
\hline 0.1 & 2801.64 & 2546.94 & 6766.11 \\
\hline 0.15 & 2939.97 & 2672.70 & 3007.16 \\
\hline 0.2 & 3158.29 & 2871.18 & 1691.53 \\
\hline 0.25 & 3491.67 & 3174.25 & 1082.58 \\
\hline 0.3 & 4008.87 & 3644.43 & 751.79 \\
\hline
\end{tabular}

\section{References}

[1] S. A. Rabboh, N. E. Bondok, T. S. Mahmoud, and H. I. El Kholy, "The Effect of Functionally Graded Materials into the Sandwich Beam Dynamic Performance," Mater. Sci. Appl., vol. 2013, no. November, pp. 751-760, 2013.

[2] C. N. Della and D. Shu, "Natural frequency of beams with embedded piezoelectric sensors and actuators," Aerospace, vol. 137, pp. 137-138, 2007.

[3] R. M. J. Groh and P. M. Weaver, "On displacement-based and mixed-variational equivalent single layer theories for modelling highly heterogeneous laminated beams," Int. J. Solids Struct., vol. 59, pp. 147-170, 2015.

[4] M. Gherlone, "On the Use of Zigzag Functions in Equivalent Single Layer Theories for Laminated Composite and Sandwich Beams: A Comparative Study and Some Observations on External Weak Layers," J. Appl. Mech., vol. 80, no. 6, p. 061004, 2013.

[5] S.-H. Schulze, M. Pander, K. Naumenko, and H. Altenbach, "Analysis of laminated glass beams for photovoltaic applications," Int. J. Solids Struct., vol. 49, no. 15-16, pp. 2027-2036, 2012.

[6] A. Dasgupta, "Eigenstrain Techniques for Modeling Adaptive Structures: II. Active Damping," J. Intell. Mater. Syst. Struct., vol. 11, no. 8, pp. 631-641, 2000.

[7] C. M. Wang, Y. Y. Zhang, and X. Q. He, "Vibration of nonlocal Timoshenko beams," Nanotechnology, vol. 18, no. 10, p. 105401, 2007.

[8] Z. Zhang, N. Challamel, and C. M. Wang, "Eringen's small length scale coefficient for buckling of nonlocal Timoshenko beam based on microstructured beam model," J. Appl. Phys., vol. 114, no. 11, 2013. 
[9] A. J. Beveridge, M. A. Wheel, and D. H. Nash, "A higher order control volume based finite element method to predict the deformation of heterogeneous materials," Comput. Struct., vol. 129, no. 0, pp. 54-62, 2013.

[10] A. J. Beveridge, M. A. Wheel, and D. H. Nash, "The micropolar elastic behaviour of model macroscopically heterogeneous materials," Int. J. Solids Struct., vol. 50, no. 1, pp. 246-255, 2013.

[11] A. R. Dehkordi, "3D Finite Element Cosserat Continuum Simulation of Layered Geomaterials," PhD Thesis, p. $263,2008$.

[12] J. C. Frame, "A computational and experimental investigation into the micropolar elastic behaviour of cortical bone," PhD Thesis_University Strat., pp. 1-199, 2013.

[13] A. Riahi and J. H. Curran, "Full 3D finite element Cosserat formulation with application in layered structures," Appl. Math. Model., vol. 33, no. 8, pp. 3450-3464, 2009.

[14] A. Waseem, A. J. Beveridge, M. A. Wheel, and D. H. Nash, "The influence of void size on the micropolar constitutive properties of model heterogeneous materials," Eur. J. Mech. A/Solids, vol. 40, pp. 148-157, 2013.

[15] M. a. Wheel, J. C. Frame, and P. E. Riches, "Is smaller always stiffer? On size effects in supposedly generalised continua," Int. J. Solids Struct., vol. 67-68, pp. 84-92, 2015.

[16] S. . R. S. L. Nakamura, "Finite element analysis of Saint-Venant end effects in micropolar elastic solids," Adapt. from Eng. Comput. 12, 571-587, pp. 1-10, 1995.

[17] R. Lakes, "Experimental methods for study of Cosserat elastic solids and other generalized elastic continua," Contin. Model. Mater. with Micro-structure, no. 1, pp. 1-22, 1996.

[18] A. J. Beveridge, M. A. Wheel, and D. H. Nash, "The micropolar elastic behaviour of model macroscopically heterogeneous materials," Int. J. Solids Struct., vol. 50, no. 1, pp. 246-255, 2013.

[19] M. McGregor and M. A. Wheel, "On the coupling number and characteristic length of micropolar media of differing topology," Proc. R. Soc. A Math. Phys. Eng. Sci., vol. 470, no. 2169, pp. 20140150-20140150, 2014.

[20] Z. Zhang, C. M. Wang, and N. Challamel, “Eringen's Length-Scale Coefficients for Vibration and Buckling of Nonlocal Rectangular Plates with Simply Supported Edges," J. Eng. Mech., vol. 141, no. 2, p. 04014117, 2015.

[21] H. Abadikhah and P. D. Folkow, "A hierarchy of dynamic equations for micropolar plates," J. Sound Vib., vol. 357, pp. 427-436, 2015.

[22] C. A. Felippa, Q. Guo, and K. C. Park, “Mass Matrix Templates: General Description and 1D Examples," Arch. Comput. Methods Eng., vol. 22, no. 1, pp. 1-65, 2015. 


\section{List of Figure}

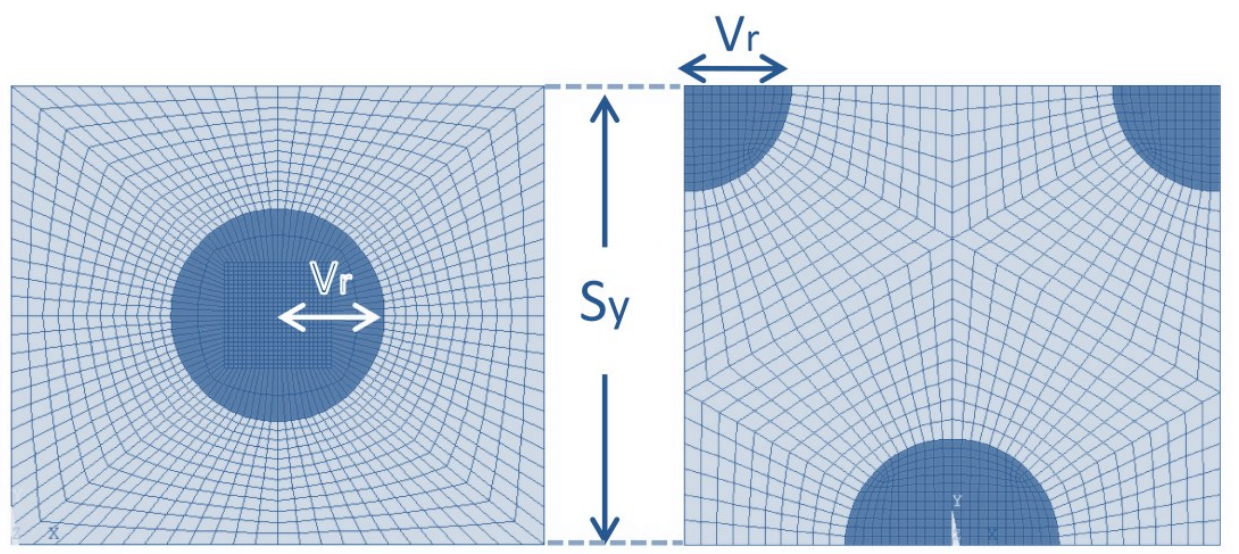

Figure 1: Unit cell consisting of two isotropic materials $r=0.2 \mathrm{~mm}$; the unit cell on the left used in generating beams with continuous surfaces and the unit cell on the right is used for when inclusions intercept the surface of the beam.
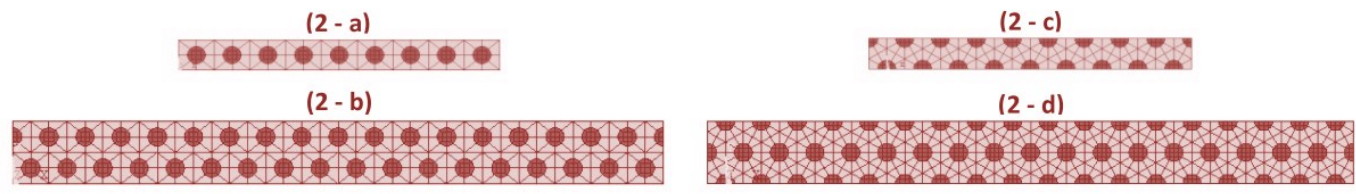

Figure 2: Models showing the arrangement of unit cells in beams; a) Beam size 1 with 9 unit cells with continuous boundaries (top, left); b) Beam size 2 with 18 unit cells in length with continuous boundaries (bottom, left); c) Beam size 1 with 9 unit cells with textured boundaries (top, right); d) Beam size 2 with 18 unit cells in length with textured boundaries (bottom, right)

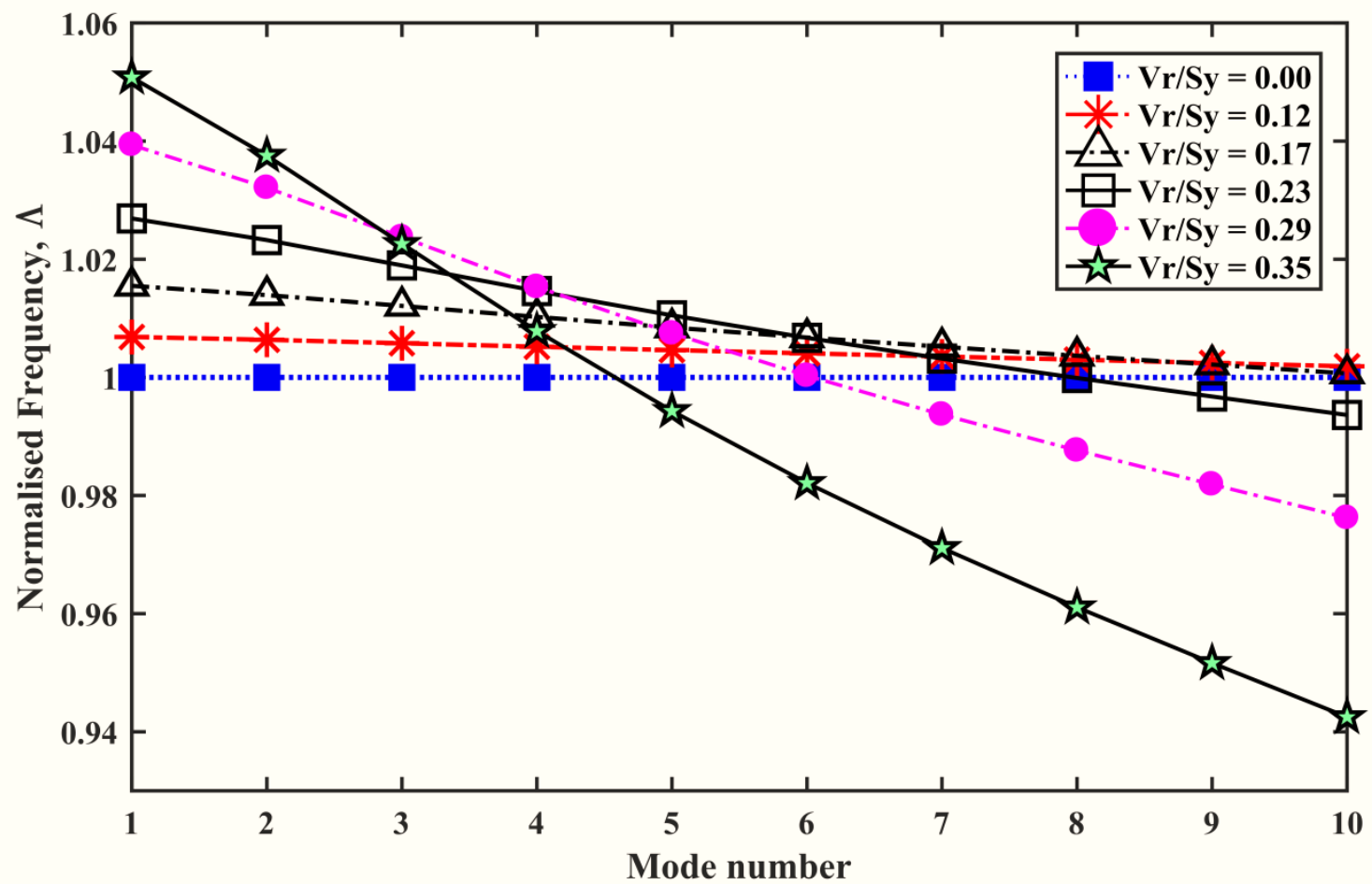


Figure 3: First ten normalised bending modal frequencies of the second smallest beam sample with voids and continuous boundaries.

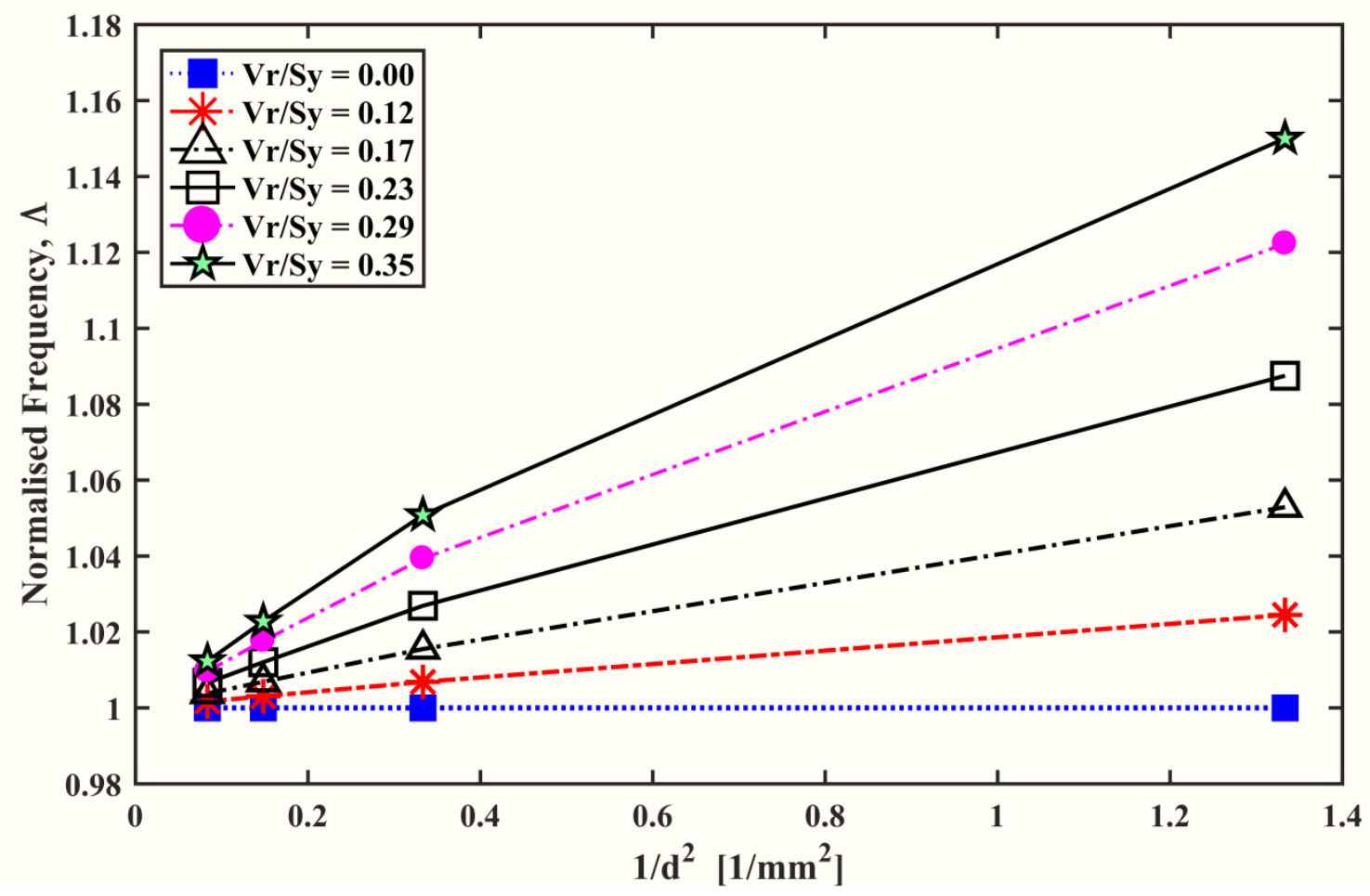

Figure 4: Normalised primary bending modal frequency of four beam sizes for beams with voids and continuous boundaries.

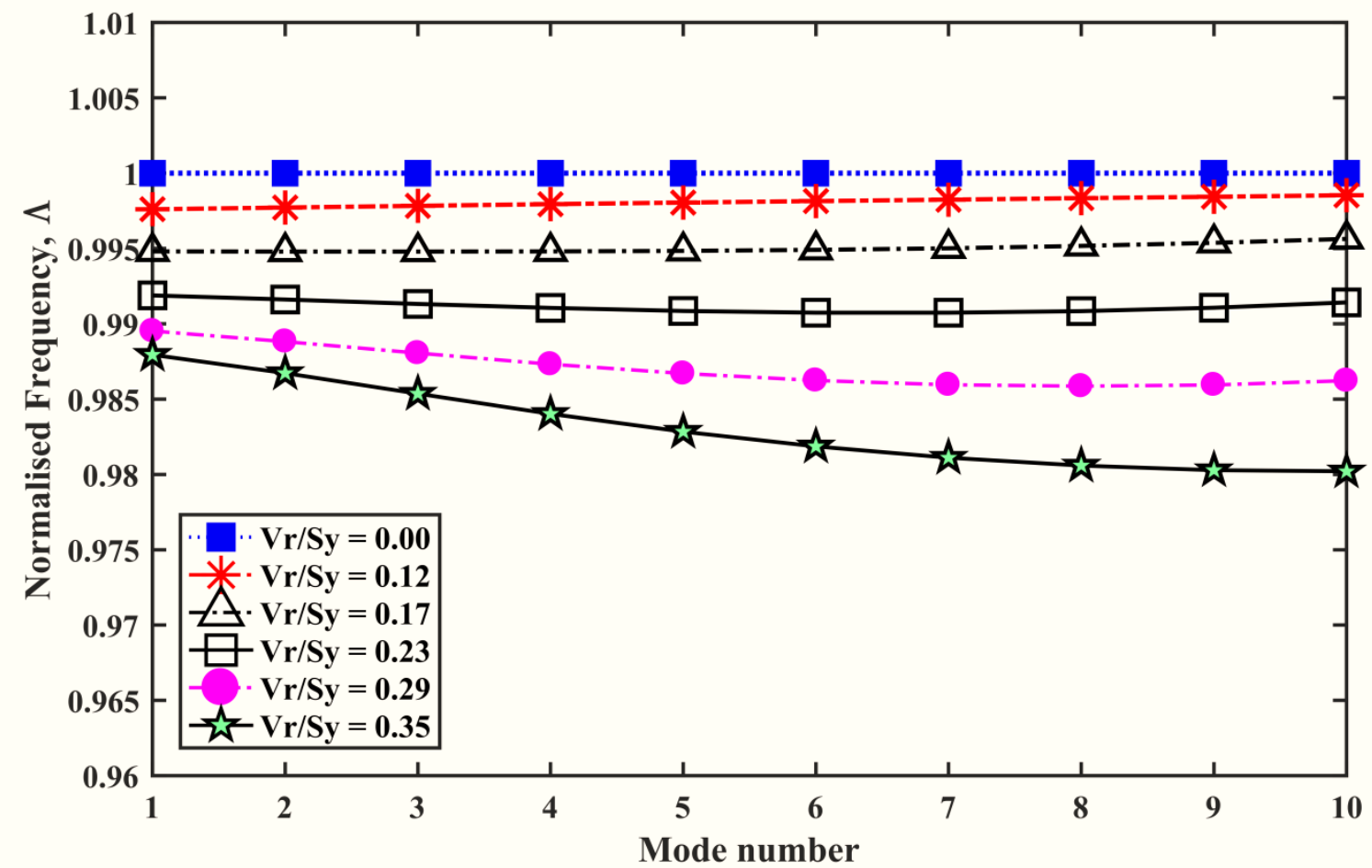

Figure 5: First ten normalised bending modal frequencies of the second smallest beam sample with compliant matrix and continuous boundaries. 


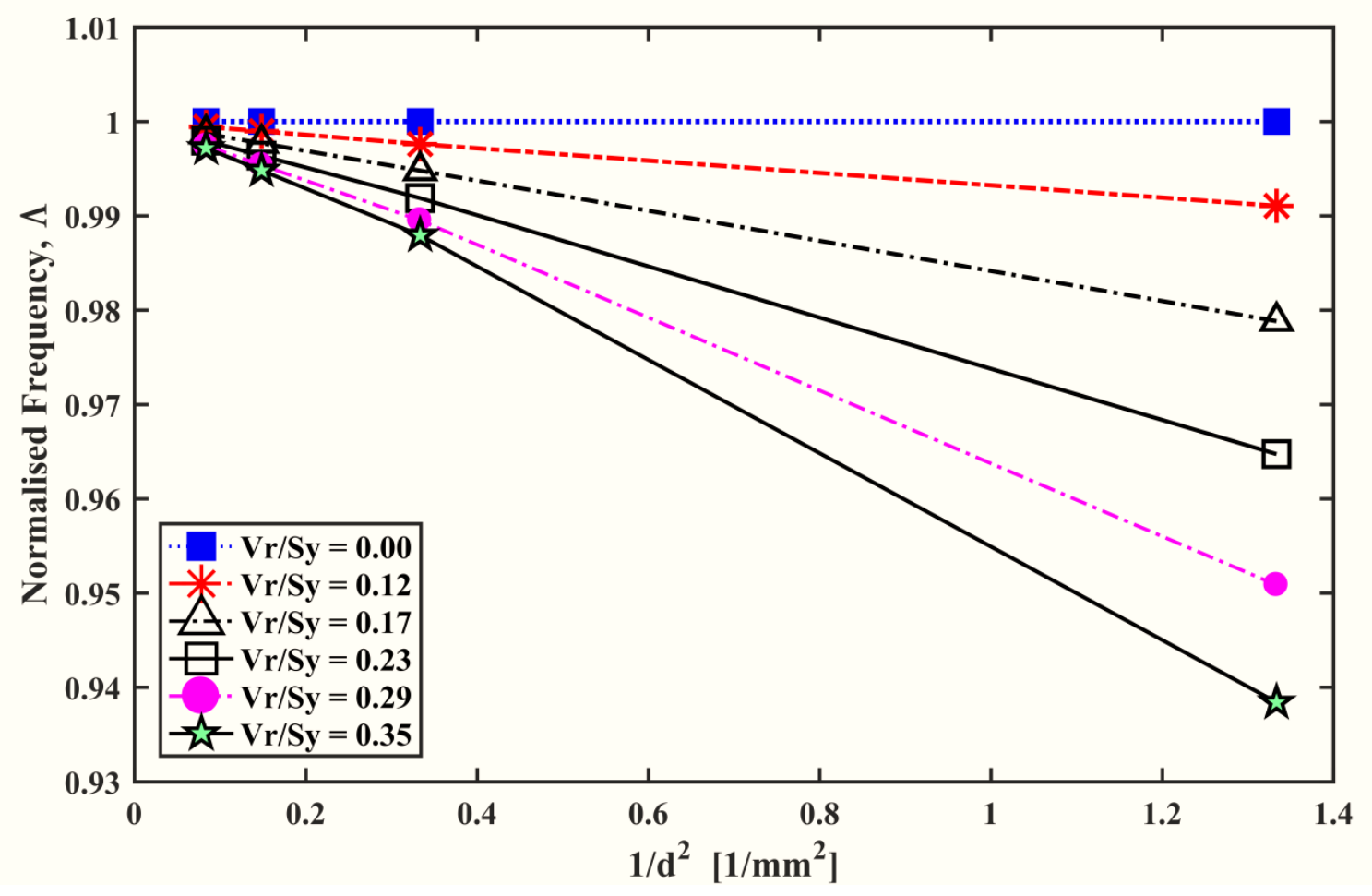

Figure 6: Normalised primary bending modal frequency of four beam sizes for beams with compliant matrix and continuous boundaries.
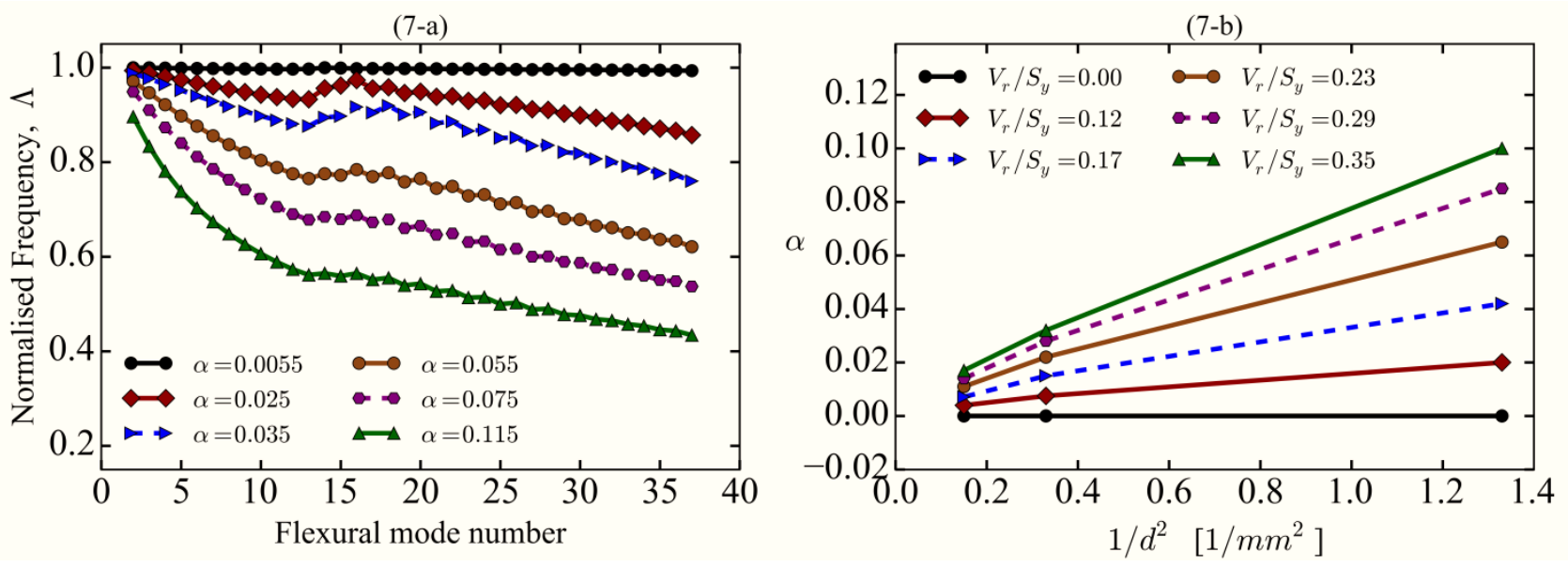

Figure 7: Results for perforated beam models with continuous boundaries and F-F boundary conditions using NLT beam theory; a) Normalised non-local Timoshenko frequency parameters for various $\alpha$ 's (graph on the left); b) Scale Coefficient ' $\alpha$ ' (Alpha), Obtained by curve fitting FE results with NLT and $\mathrm{Vr} / \mathrm{Sy}$ is the normalised void radius changing from 0 to 0.35 (graph on the right).

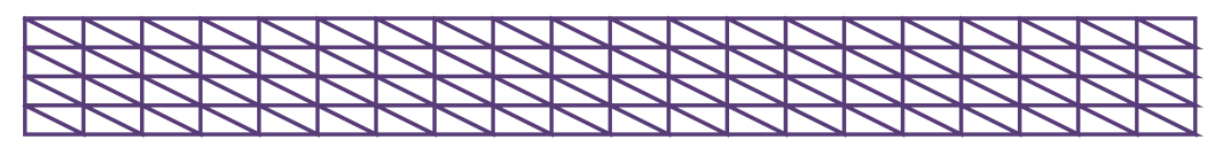

Figure 8: Representation of CVFEM meshed beam model 

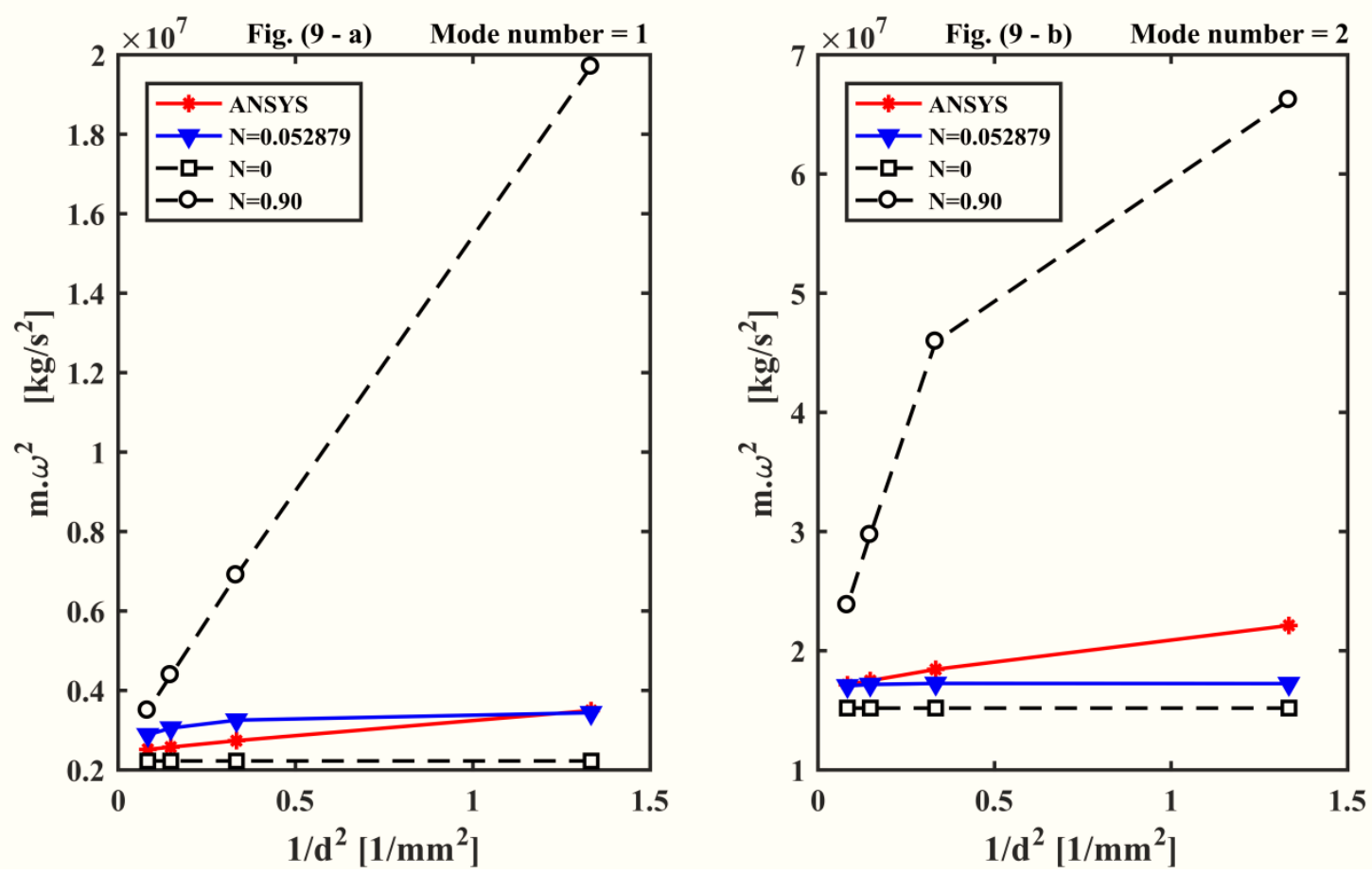

Figure 9: The variation of $\mathrm{m} \omega^{2}$ with beam size for beams with compliant inclusions and continuous surface and volume fraction equal to 0.23 ; a) The variation of $m \omega^{2}$ at flexural mode 1 (on the left); b) The variation of $m \omega^{2}$ at flexural mode 2 (on the right).

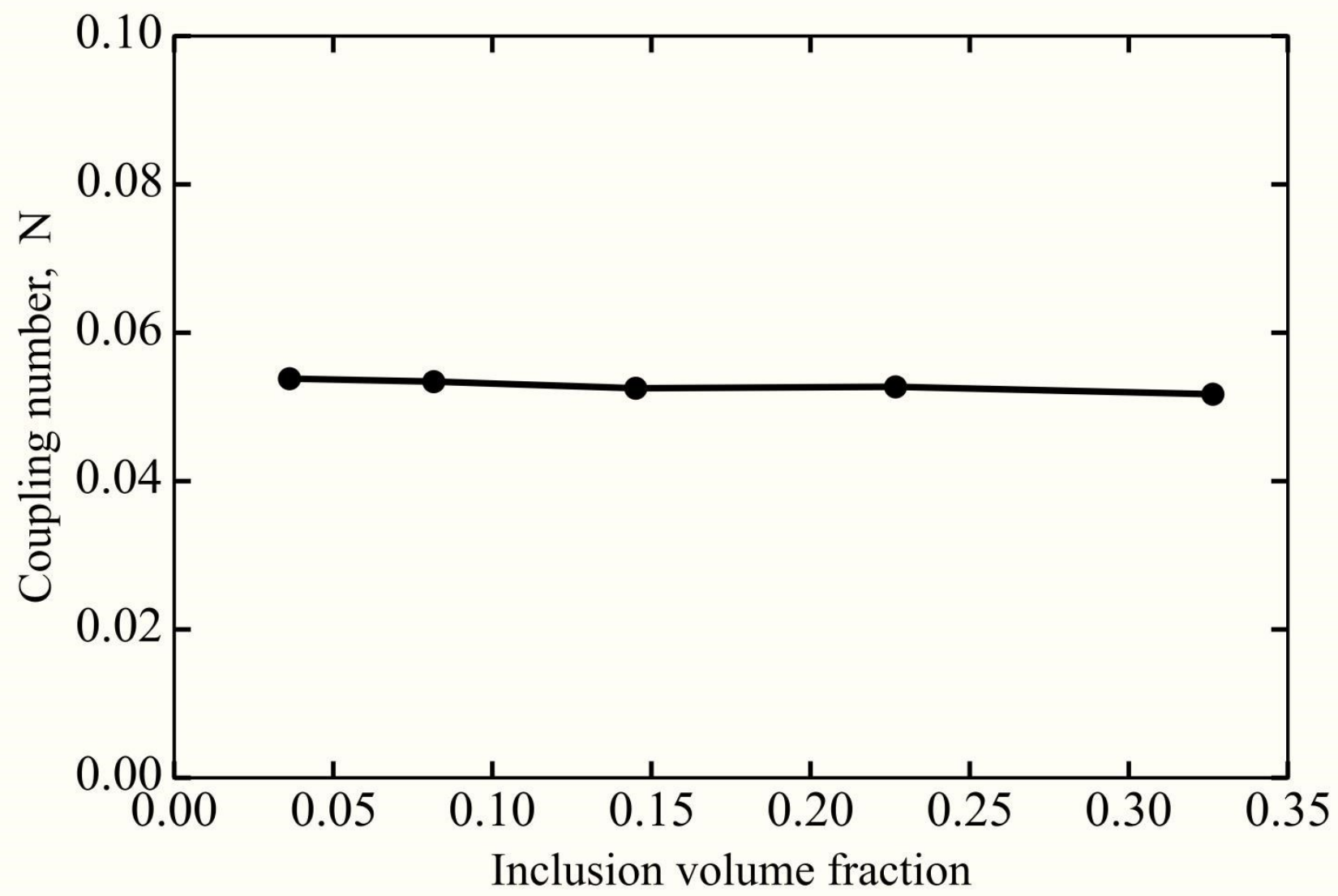

Figure 10: Values for coupling number, N, vs. inclusions volume fraction for beams with compliant inclusions and continuous boundaries 


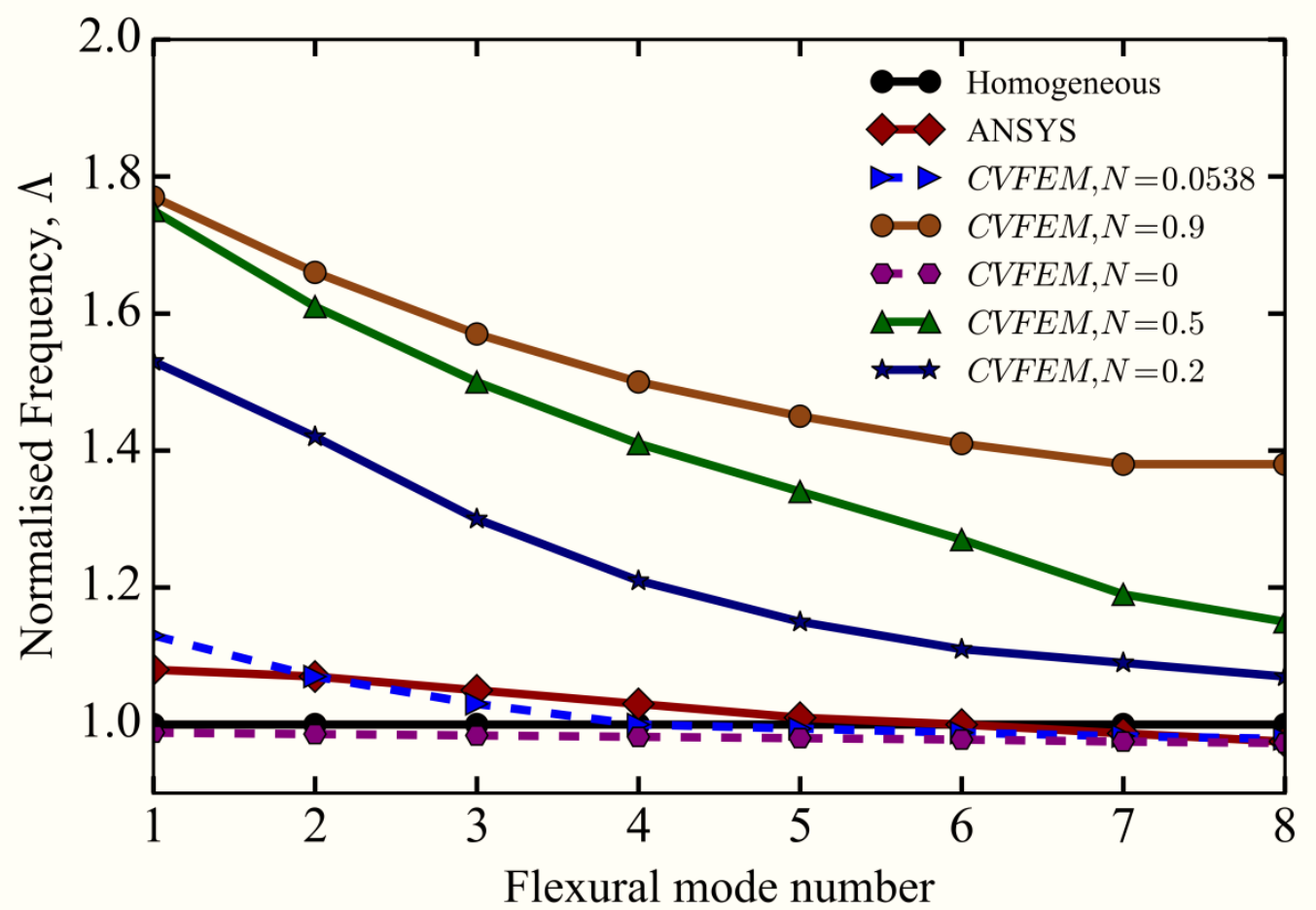

Figure 11: Normalised flexural modal frequencies produced by ANSYS squared markers and CVFEM code shown in dotted line and triangular markers. $\mathbf{l} \mathbf{c}=\mathbf{0 . 6 5 2 2} \mathbf{~} \mathbf{m m}, \mathbf{N}=\mathbf{0 . 0 5 3 8}, \mathrm{d}=1.732 \mathrm{~mm}$ and the void volume fraction is 0.23

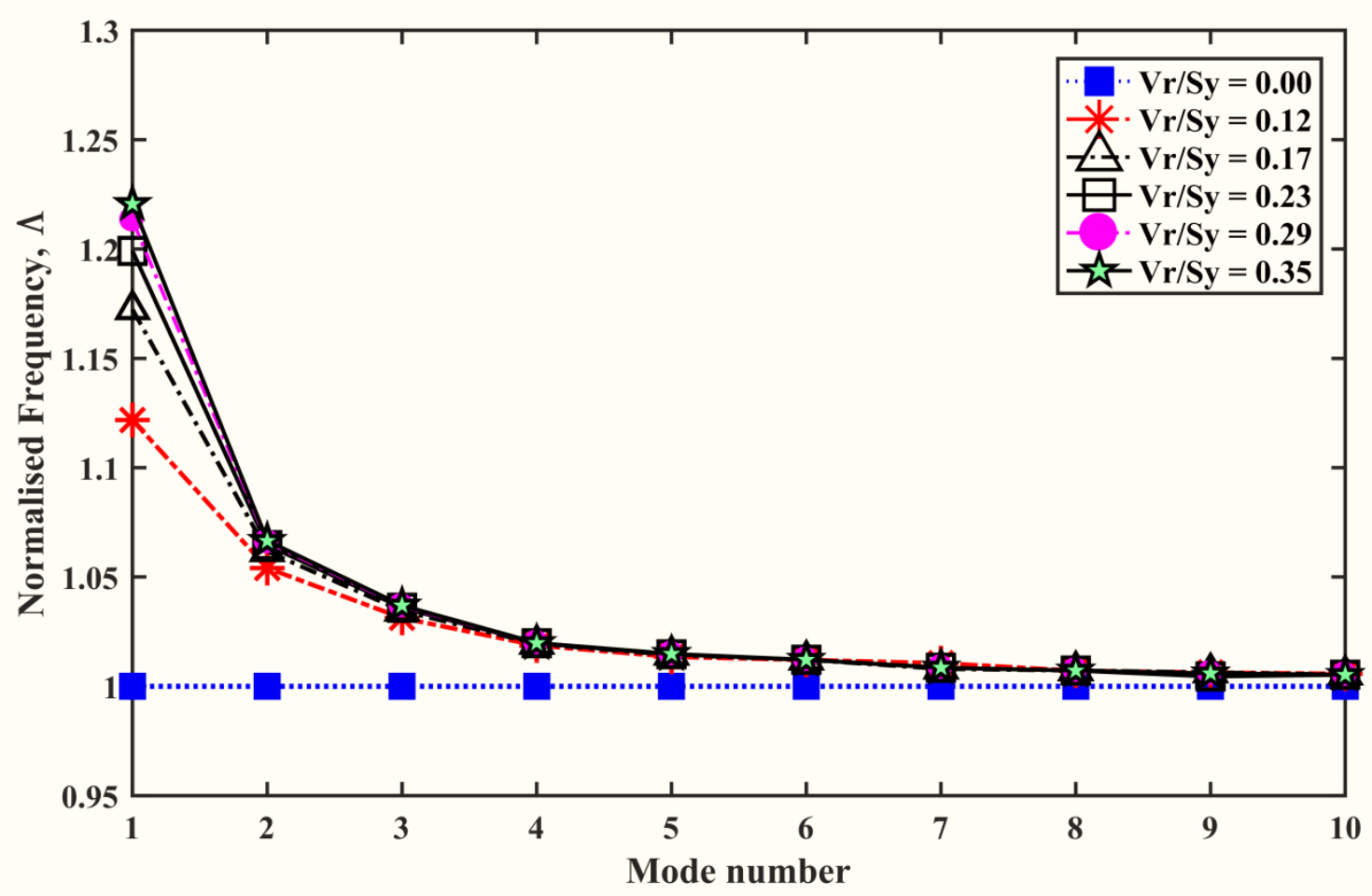

Figure 12: Normalised flexural modal frequencies of the second smallest beam sample with voids and continuous boundaries 


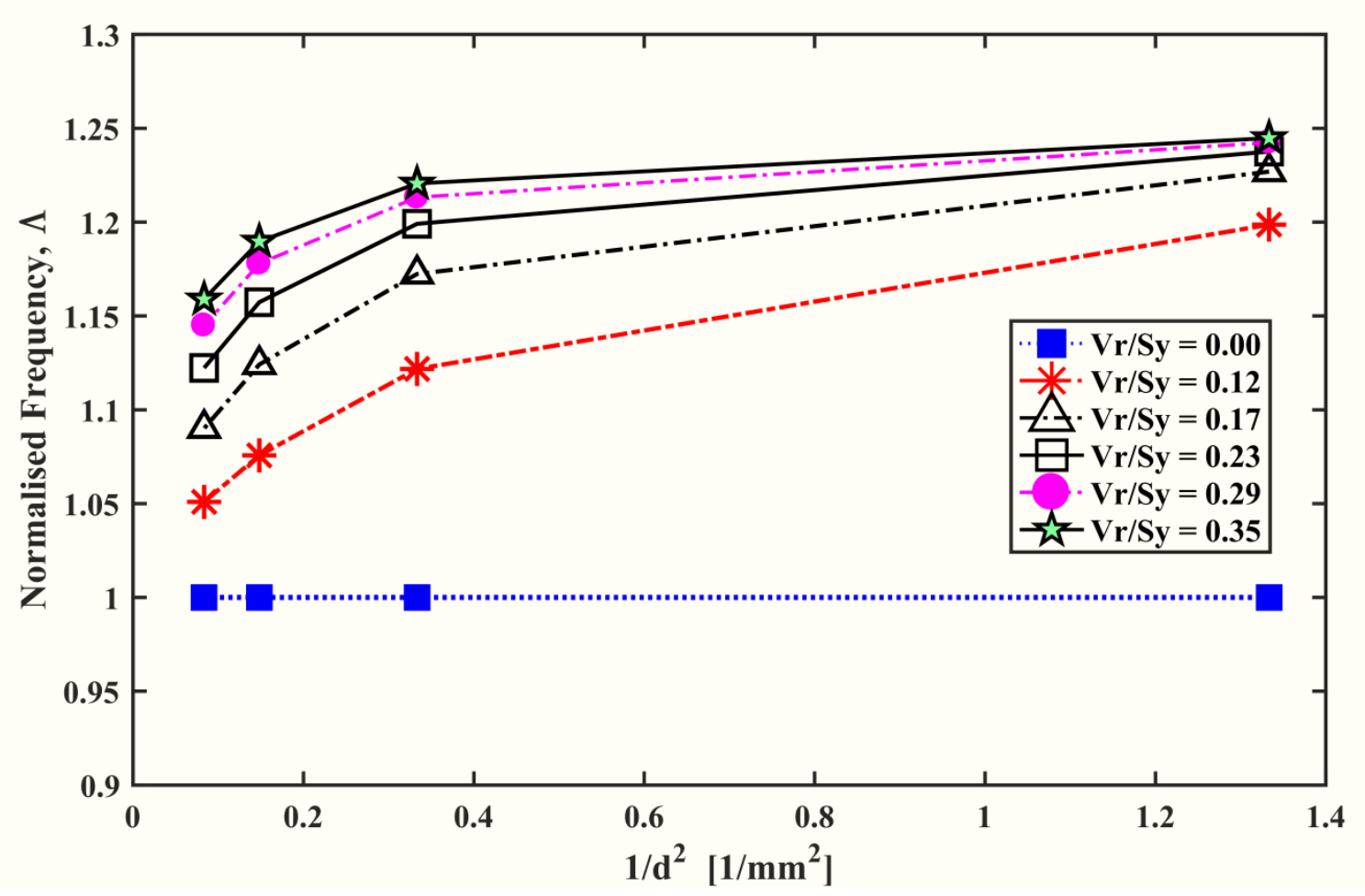

Figure 13: Normalised flexural modal frequencies of the first mode for beams with voids and continuous boundaries 